\title{
Spectral evolution of the nova V2468 Cygni: asymmetric and variable profiles of its emission lines ${ }^{\star}$
}

\author{
T. Iijima ${ }^{1}$ and H. Naito ${ }^{2,3}$ \\ 1 Astronomical Observatory of Padova, Asiago Section, Osservatorio Astrofisico, 36012 Asiago (Vi), Italy \\ e-mail: takashi.iijima@oapd.inaf.it \\ 2 Nishi-Harima Astronomical Observatory, Sayo-cho, 679-5313 Hyogo, Japan \\ 3 Graduate School of Science, Nagoya University, Furo-cho, Chikusa-ku, 464-8602 Nagoya, Japan
}

Received 22 July 2010 / Accepted 28 October 2010

\section{ABSTRACT}

\begin{abstract}
The spectral evolution of the classical nova V2468 Cygni (Nova Cyg 2008) was monitored at Asiago Astrophysical Observatory in Italy and at Nishi-Harima Astronomical Observatory in Hyogo Japan from soon after the discovery in March 2008 to the end of 2009. The notable peculiarities in the spectra of this nova were the asymmetric and variable profiles of its emission lines. The emission-line profiles of $\mathrm{H}$ I varied in the early decline stage in 2008, while their profiles were nearly stable in 2009. On the other hand, large variations in the profiles of the emission lines of [O III], [Fe VII], and He II were observed in 2009. In the spectrum obtained on 2009 October 15, the red parts of the emission lines of [O III] strengthened with respect to the profiles in September, while the red parts of the lines of He II and [Fe VII] faded. The emission lines of [Fe VII] 5721 and [Fe VII] + [Ca V] 6087 exhibited convex profiles on 2009 September 27 and 28 in a similar way to the other lines, while only these lines had concave profiles on October 26 and 29 . It seems to be difficult to explain the variations in the emission-line profiles with any known model of nova shells. The absolute magnitude at light maximum and the distance to the nova are estimated to be $M_{V}=-8.8 \pm 0.3 \mathrm{mag}$ and $d=5.5 \pm 0.8 \mathrm{kpc}$. Using the intensities of H I, He I, and He II lines observed in September 2009, we estimated the helium abundance in number to be $N(\mathrm{He})=0.20 \pm 0.01$, and the mass of the ejecta to be $1.7 \pm 1 \times 10^{-5} M_{\odot}$. A very broad emission line from $3790 \AA$ to $3850 \AA$ was detected in the spectrum obtained on 2009 July 21. We propose that the multiple lines of [Fe V] 3F, 3839.3, 3794.9, 3851.3, 3819.9 etc. suddenly strengthened at that time, but the cause of this phenomenon is not known.
\end{abstract}

Key words. stars: individual: V2468 Cygni - novae, cataclysmic variables

\section{Introduction}

The nova V2468 Cygni (Nova Cyg 2008) was discovered by Kaneda on 2008 March 7.8 UT as a star of 8.2 mag in unfiltered CCD frames (Nakano 2008). Low dispersion spectra obtained soon after its discovery contained prominent emission lines of $\mathrm{H}$ I and Fe II accompanied by P Cygni type absorption components (Beaky 2008; Nogami et al. 2008). Rudy et al. (2008) obtained infrared spectra with the Infrared Telescope Facility on 2008 March 13 and April 12. They found rich emission lines of C I, N I, O I, Fe II, and Ca II on March 13, then the emission lines of $\mathrm{Ca}$ II faded and prominent emission lines of He I were detected on April 12 (Rudy et al. 2008). They estimated the interstellar extinction to the nova to be $E(B-V)=0.77$ from the intensity ratio of the infrared O I lines (Rudy et al. 2008). Schwarz et al. (2009) detected X-rays from this nova by the Swift observations in June and August 2009. They also carried out some UV and optical observations in the same period (Schwarz et al. 2009).

The spectral evolution of this nova was monitored at Asiago Astrophysical Observatory of the University of Padova in Italy and at Nishi-Harima Astronomical Observatory in Hyogo Japan. In this paper, we report the spectral evolution of this nova from soon after the discovery to the end of 2009. We discuss mainly the peculiar asymmetric and variable profiles of the emission lines. Many classical novae showed variable profiles

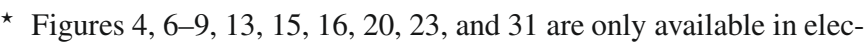
tronic form at http://www . aanda.org
}

of the emission lines in the early decline stage. However, the profiles of the emission lines of this nova were also variable at the nebular stage.

\section{Observations}

The medium dispersion spectroscopy at Asiago Observatory was carried out using a Boller \& Chivens grating spectrograph mounted on the $122 \mathrm{~cm}$ telescope at Asiago. The spectral resolution was $R=\lambda / \Delta \lambda \cong 1000$ with a grating of 600 lines $\mathrm{mm}^{-1}$. Two high dispersion spectra $(R \cong 10000)$ were obtained with a Reosc echelle spectrograph mounted on the $182 \mathrm{~cm}$ telescope at the Mount Ekar station of the Astronomical Observatory of Padova.

The spectrograph MALLS (Medium And Low dispersion Long slit Spectrograph) mounted on the $200 \mathrm{~cm}$ Nayuta telescope was used for the observations at Nishi-Harima Astronomical Observatory. A grating of 300 lines $\mathrm{mm}^{-1}$ was used for the medium dispersion spectra $(R \cong 900)$, and one high dispersion spectrum $(R=6000)$ was obtained on 2008 March 11 with a grating of 1800 lines $\mathrm{mm}^{-1}$. A log of the observations is given in Table 1 and the dates of the observations are indicated by vertical bars in Fig. 1 .

The spectra were reduced at the respective observatories using the standard tasks of the NOAO IRAF ${ }^{1}$ package. The

\footnotetext{
1 IRAF is distributed by NOAO for Research in Astronomy, Inc. under cooperative agreement with the National Science Foundation.
} 
Table 1. Log of spectroscopic observations of V2468 Cyg.

\begin{tabular}{|c|c|c|c|c|c|}
\hline$\overline{\text { Date }}$ & $\overline{\overline{\text { UT }}}$ & 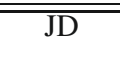 & $\begin{array}{r}\text { Exp. } \\
\text { s }\end{array}$ & Inst. & $\begin{array}{c}\begin{array}{c}\text { Range } \\
\mathrm{nm}\end{array} \\
\text {. }\end{array}$ \\
\hline 2008 & & & & & \\
\hline March 8 & $18: 42$ & 4534.29 & 1800 & MALLS & $405-672$ \\
\hline March 10 & $18: 57$ & 4536.30 & 1800 & " & \\
\hline March 11 & $18: 42$ & 4537.29 & 1800 & " & " \\
\hline March 11 & $19: 57$ & 4537.34 & 1800 & " & $571-613$ \\
\hline March 12 & 4:05 & 4537.67 & 60 & $B \& C$ & $340-580$ \\
\hline March 12 & 4:08 & 4537.68 & 600 & " & "' \\
\hline March 12 & $18: 14$ & 4538.27 & 1800 & MALLS & $405-672$ \\
\hline March 13 & $4: 21$ & 4538.68 & 60 & $\mathrm{~B} \& \mathrm{C}$ & 453-694 \\
\hline March 13 & $4: 24$ & 4538.68 & 600 & & \\
\hline March 15 & $17: 39$ & 4541.25 & 1800 & MALLS & $405-672$ \\
\hline March 18 & $4: 18$ & 4543.69 & 900 & $\mathrm{~B} \& \mathrm{C}$ & $338-576$ \\
\hline March 23 & $3: 22$ & 4548.65 & 60 & " & $522-765$ \\
\hline March 23 & $3: 24$ & 4548.65 & 600 & " & " \\
\hline March 23 & $4: 15$ & 4548.68 & 900 & " & $330-568$ \\
\hline April 20 & $2: 38$ & 4576.62 & 1800 & Ech & $435-612$ \\
\hline April 28 & $1: 53$ & 4584.59 & 2400 & " & 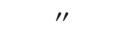 \\
\hline July & $14: 35$ & 4656.15 & 3000 & MALLS & $407-674$ \\
\hline July & $14: 31$ & 4672.13 & 3600 & " & $408-675$ \\
\hline July & $14: 59$ & 4672.14 & 1800 & " & \\
\hline August 9 & $14: 46$ & 4688.14 & 3600 & " & 1 \\
\hline Oct. & $12: 27$ & 4749.04 & 3600 & " & $407-674$ \\
\hline $\begin{array}{c}\text { Nov. } 13 \\
2009\end{array}$ & $9: 26$ & 4783.91 & 3000 & " & $406-673$ \\
\hline June & $0: 52$ & 4985.56 & 3600 & $B \& C$ & $336-575$ \\
\hline July & $1: 18$ & 5033.57 & 3000 & " & $340-567$ \\
\hline Sep. & $21: 43$ & 5102.41 & 600 & " & $566-808$ \\
\hline Sep. & $21: 54$ & 5102.43 & 2400 & " & 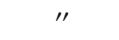 \\
\hline Sep. & $21: 33$ & 5103.42 & 3000 & " & $340-579$ \\
\hline Oct. & $22: 45$ & 5120.46 & 2400 & " & $350-588$ \\
\hline Oct. 16 & $22: 34$ & 5121.45 & 1800 & " & $553-795$ \\
\hline Oct. & $20: 27$ & 5131.37 & 3000 & " & $350-588$ \\
\hline Oct. & $20: 36$ & 5134.37 & 1800 & I & $560-801$ \\
\hline
\end{tabular}

Notes. JD: Julian date -2450000 . UT: Universal time at start of exposure.

spectrophotometric calibrations were performed using the spectra of the standard star HD 192281 obtained during the same nights of the observations.

\section{Interstellar extinction and distance}

Figure 1 shows the $V$ light curve of this nova observed by the members of VSNET Japan. The data around light maximum are shown in the upper right corner of the figure. We estimated the magnitude and the epoch of light maximum to be $m_{V}=7.3 \mathrm{mag}$ at 2008 March 8.3 UT $(\mathrm{JD}=2454533.8)$. The decline rate at two magnitudes was estimated to be $7.8 \pm 0.5$ days. The relation between the decline rate and the absolute magnitude at light maximum (Della Valle \& Livio 1995) gives $M_{V}=-8.8 \pm 0.3 \mathrm{mag}$.

The high dispersion spectra showed narrow interstellar absorption components of Na I D1 and D2, which were superimposed on a broad emission feature. The equivalent widths of the interstellar absorption components were $0.62 \pm 0.03 \AA$ for D1 and $0.73 \pm 0.03 \AA$ for $\mathrm{D} 2$. These values are the means of the equivalent widths measured on the three available high dispersion spectra.

The column density of sodium atoms was estimated to be $\log (N(\mathrm{Na}) \cdot L)=13.3 \pm 0.1$ atoms $\mathrm{cm}^{-2}$ using the doublet ratio method of Münch (1968). The chemical abundance of the interstellar matter is $\log (N(\mathrm{Na}) / N(\mathrm{H}))=-8.4($ Cohen 1975), and

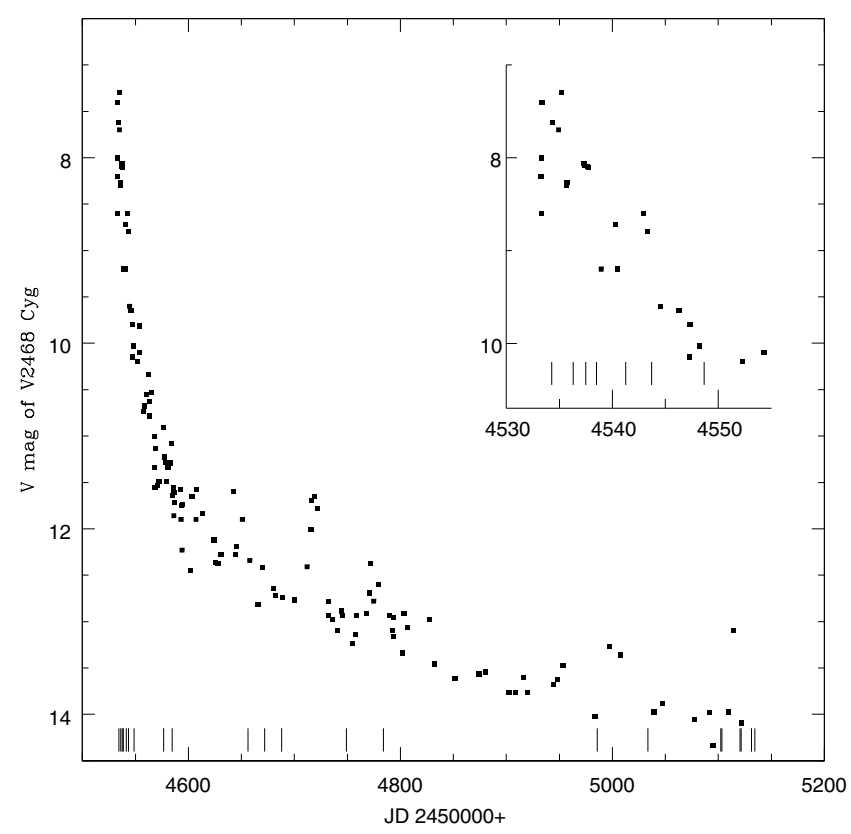

Fig. 1. The $V$ light curve of V2468 Cyg. The data around light maximum are shown in the upper right corner. The dates of the spectroscopic observations are indicated by vertical bars.

the relation between the column density of hydrogen atoms and the interstellar extinction is $N(\mathrm{H}) \cdot L / E(B-V)=6.2 \times$ $10^{21}$ atoms cm $\mathrm{cm}^{-1}$ (Jenkins \& Savage 1974). Thus we inferred the amount of interstellar extinction to the nova to be $E(B-V)=0.8 \pm 0.1$. This result agrees with the value $E(B-V)=$ 0.77 derived by Rudy et al. (2008) and $E(B-V)=0.8$ derived from the Balmer decrement by Schwarz et al. (2009). Comparing the apparent and absolute magnitudes at light maximum, we estimated the distance to the nova to be $d=5.5 \pm 0.8 \mathrm{kpc}$, where we assumed $E(B-V)=0.77$, that is $A_{V}=3.1 \cdot E(B-V)=2.4 \mathrm{mag}$.

The mean radial velocity of the interstellar absorption components of Na I D1 and D2 was $+12.8 \pm 1 \mathrm{~km} \mathrm{~s}^{-1}$ in the frame of the local standard of rest. This velocity is consistent with that of the Galactic motion of the interstellar matter at the distance $5.5 \mathrm{kpc}$ in the direction of the nova (Kerr \& Westerhout 1965). The heliocentric radial velocity of the line centre of the emission line of [N II] 5755, measured in the high dispersion spectra, was $+5.6 \pm 0.6 \mathrm{~km} \mathrm{~s}^{-1}$. This velocity may reflect that of the centre of gravity of this nova system.

\section{Spectral evolution}

\subsection{Early decline stage}

Figure 2 shows a tracing of our first spectrum obtained at NishiHarima Observatory on 2008 March 8, one day after the discovery and near the epoch of light maximum. The spectrum showed prominent emission lines of H I, Fe II, and Si II accompanied by P Cygni type absorption components, which are typical spectral features of a classical nova around light maximum. Our results are consistent with those reported by Nogami et al. (2008) for spectra obtained in the same night.

The spectral features subsequently rapidly changed to those of post maximum. Figure 3 shows a tracing of the spectrum obtained on March 10, where the absorption components faded and the emission components became broad. The absorption 


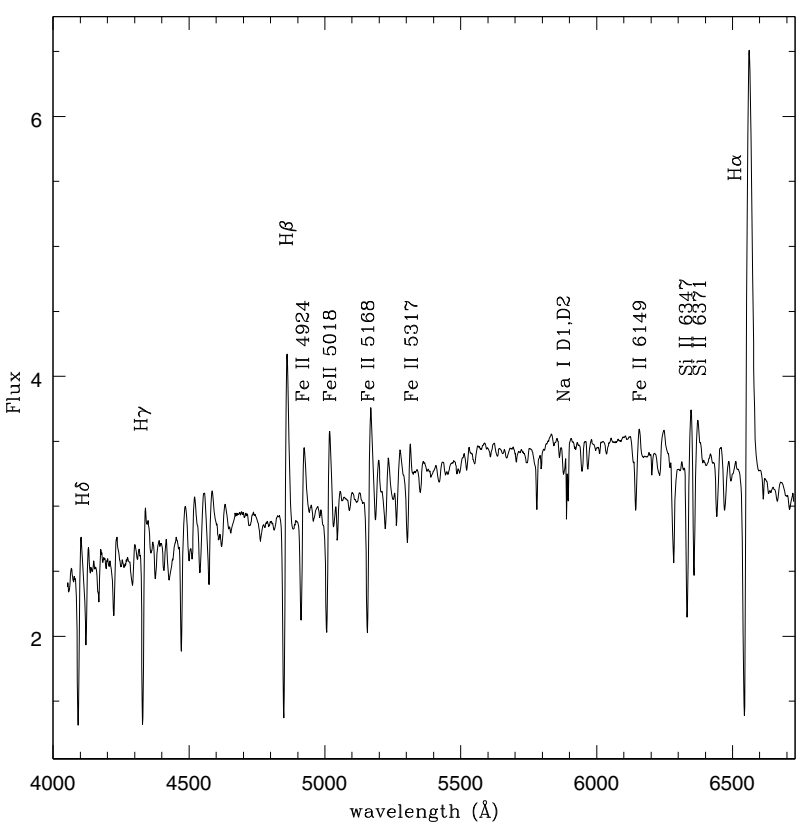

Fig. 2. A spectrum of V2468 Cyg on 2008 March 8. The unit of the ordinate is $10^{-12} \mathrm{erg} \mathrm{cm}^{-2} \mathrm{~s}^{-1} \AA^{-1}$.

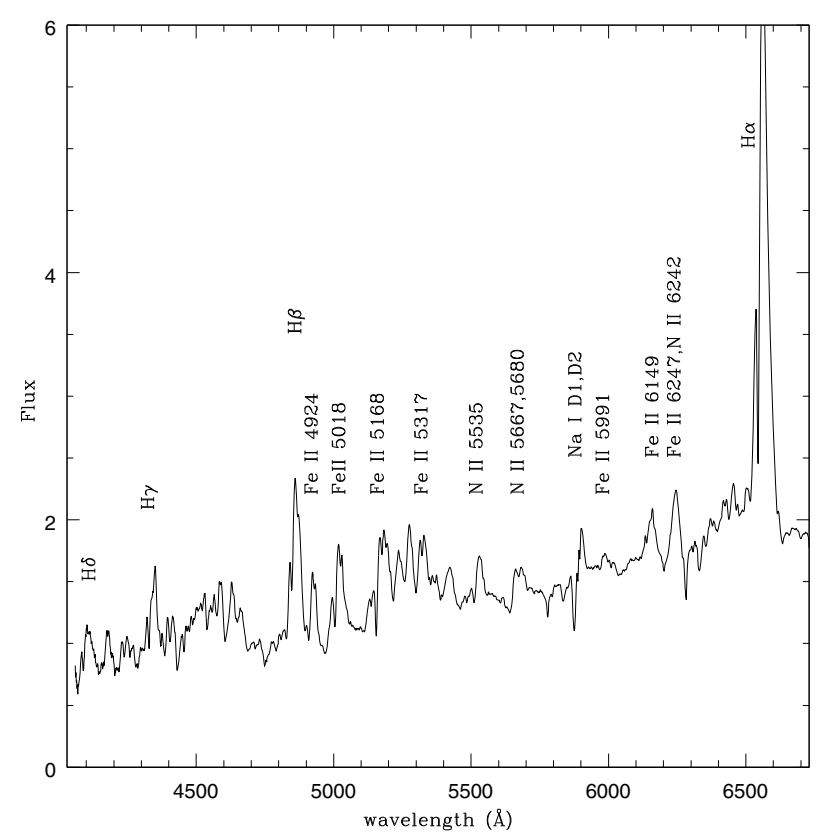

Fig. 3. A spectrum of V2468 Cyg on 2008 March 10. The unit of the ordinate is $10^{-12} \mathrm{erg} \mathrm{cm}^{-2} \mathrm{~s}^{-1} \AA^{-1}$.

components of $\mathrm{H}$ I lines were visible, but with their central depths above the continuum level. The prominent emission lines detected first in this spectrum were N II 3, 5667, 5680, N II 63, 5535, Na I D1 + D2, and an emission line at $5990 \AA$, which was identified as Fe II 46, 5991. On the other hand, the emission components of Si II lines disappeared.

The spectrum did not evolve in a straight forward way. The absorption components strengthened again on March 11 and 12. Figure 4 shows a tracing of a spectrum obtained on March 11,

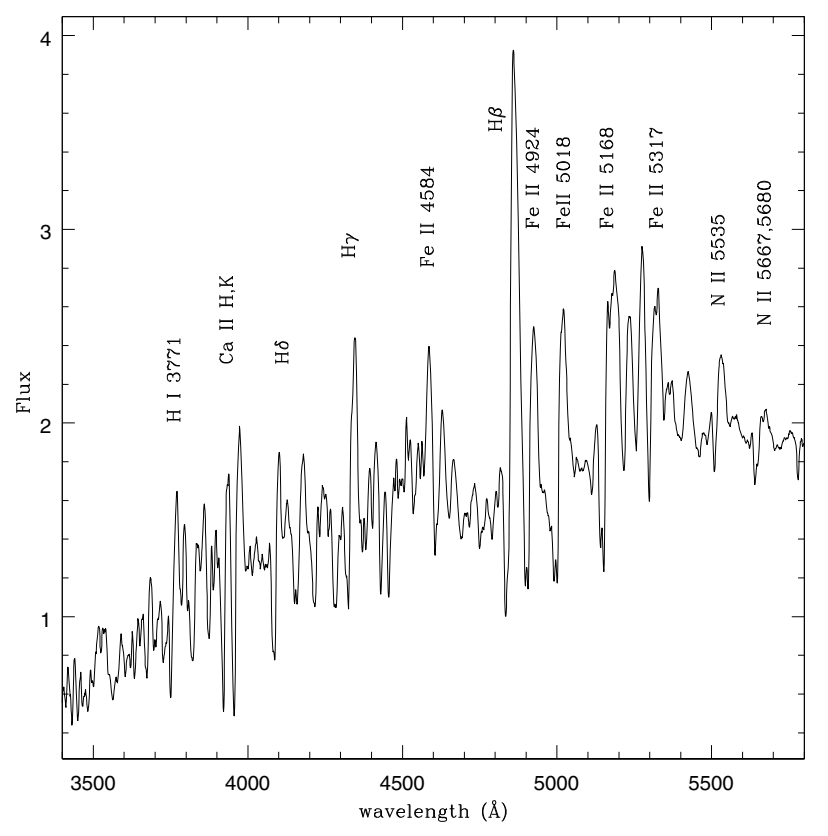

Fig. 5. A spectrum of V2468 Cyg on 2008 March 12.2. The unit of the ordinate is $10^{-12} \mathrm{erg} \mathrm{cm}^{-2} \mathrm{~s}^{-1} \AA^{-1}$.

where the absorption components of $\mathrm{H}$ I lines were blue-shifted by about $-1780 \pm 20 \mathrm{~km} \mathrm{~s}^{-1}$ and their central depths were below the continuum level.

Figure 5 shows a tracing of the first spectrum obtained at Asiago Observatory on March 12.2. The absorption components had by then become deeper. The prominent $\mathrm{Ca}$ II $\mathrm{H}$ and $\mathrm{K}$ emission lines were accompanied by absorption components blueshifted by $-1340 \pm 10 \mathrm{~km} \mathrm{~s}^{-1}$. The blue-shift of the main absorption component of $\mathrm{H} \beta$ was $-1750 \pm 10 \mathrm{~km} \mathrm{~s}^{-1}$ and the secondary absorption component, blue-shifted by $-1070 \pm 10 \mathrm{~km} \mathrm{~s}^{-1}$, was barely visible. The depths of the two absorption components were nearly comparable in the cases of Fe II 42, 4924, 5018, and 5168. The mean blue-shift of the higher velocity absorption components of the Fe II lines was $-1650 \pm 10 \mathrm{~km} \mathrm{~s}^{-1}$ and that of the other components was $-1070 \pm 10 \mathrm{~km} \mathrm{~s}^{-1}$.

The development of the absorption components on March 11 and 12 was a short-lived phenomenon, because the absorption components had weakened when a spectrum was obtained $14 \mathrm{~h}$ later in Japan at March 12.8 (Fig. 6), then became still weaker in the spectra obtained on March 13 at Asiago Observatory (Fig. 7). A brightening of this nova to $m_{V}=8.1 \mathrm{mag}$ was seen on March 12 (Fig. 1 and see also Munari et al. 2008). A new mass ejection probably occurred on March 11 and 12.

The absorption components were weak on March 15 (Fig. 8), and the emission lines of H I and Fe II had double peak profiles. The separations between the two peaks were about $820 \pm$ $10 \mathrm{~km} \mathrm{~s}^{-1}$. A detailed discussion of these profiles is given in Sect. 5.1. The emission line of [O I] 5577 was first noticed in this spectrum (Fig. 8).

The absorption components of $\mathrm{H} \mathrm{I}$ and Fe II lines developed again on March 18 (Fig. 9). The spectral feature of March 18 was similar to those of March 11 (Fig. 4) and March 12 (Fig. 5). A brightening to $m_{V}=8.6 \mathrm{mag}$ was observed on March 17.4 (Fig. 1 and see also IAU Circ. 8928). A small mass ejection may have occurred at that time. The mean blue-shift of the higher velocity absorption components was $-2120 \pm 10 \mathrm{~km} \mathrm{~s}^{-1}$ and that 


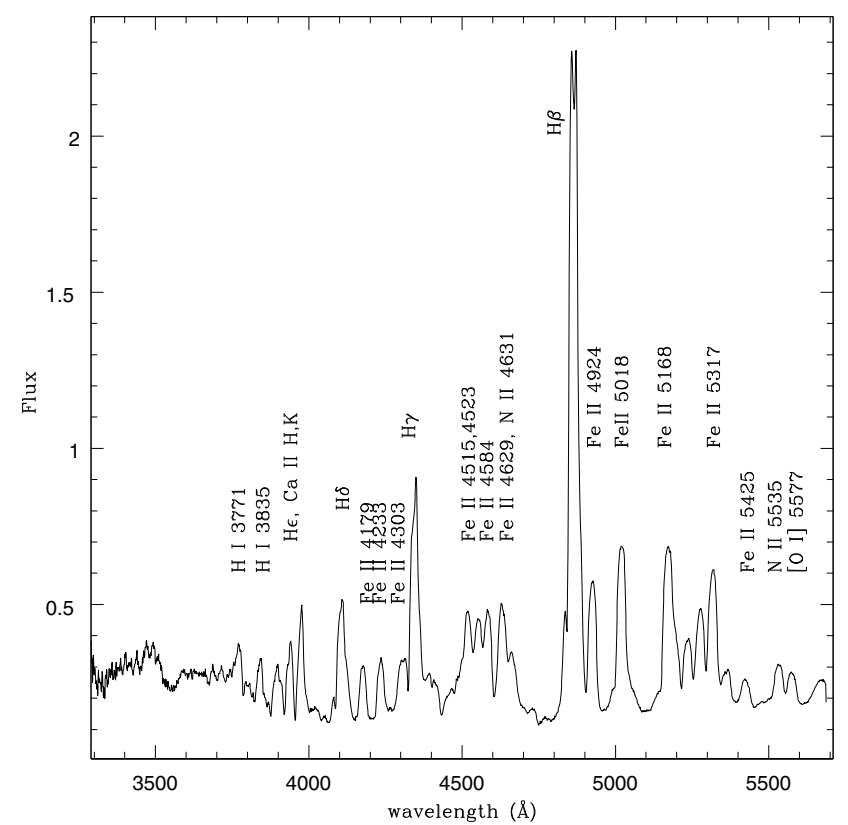

Fig. 10. A blue spectrum of V2468 Cyg on 2008 March 23. The unit of the ordinate is $10^{-12} \mathrm{erg} \mathrm{cm}^{-2} \mathrm{~s}^{-1} \AA^{-1}$.

of the lower velocity ones was $-1270 \pm 10 \mathrm{~km} \mathrm{~s}^{-1}$. A weak trace of the emission line [N II] 5755 was detected in this spectrum (Fig. 9).

The tracings of the blue and red spectra obtained on March 23, 15 days after and 2.6 mag below light maximum, are shown in Figs. 10 and 11. The profile of $\mathrm{H} \alpha$ is shown in Fig. 11 by one twentieth scale. The absorption components of all lines weakened and the double peak profiles appeared again. The emission lines of [O I] 5577 and [N II] 5755 largely strengthened and that of [O I] 6300 was detected (Fig. 11). The weak emission features at $6715 \AA$ and $7111 \AA$ could not be identified (Sect. 7.1).

\subsection{Nebular stage}

Because of unfavourable weather, we were unable to obtain any medium dispersion spectrum in April, May, and June 2008 in either Italy or Japan. When a new spectrum was obtained on 2008 July 8, 122.4 days after and 5.1 mag below light maximum, the nova was entering the nebular stage. As seen in Fig. 12, the emission lines of [O III] appeared, but their intensities relative to $\mathrm{H} \beta$ were much lower than those observed later. The emission line of He II 4686 was weak. The prominent emission lines in the red region were of $\mathrm{H} \alpha$, [N II] 5755, He I 5876, 6678, N II 5667, 5680, and [O I] 6300.

The ionisation level of the spectrum rose rapidly. Figure 13 shows a tracing of the spectrum obtained on July 24, where the emission line of He II 4686 could be clearly seen. The emission lines of [O III] strengthened with respect to $\mathrm{H}$ I lines, and the line at $6080 \AA$ was identified as a blend of [Fe VII] 6087 and [Ca V] 6087.

The spectrum obtained on 2008 August 8 showed nearly the same spectral features as on July 24. The tracings of the spectra obtained on 2008 October 9 and November 13 are shown in Figs. 14 and 15. Intensities of selected emission lines in these spectra were measured and the results are presented in Table 2

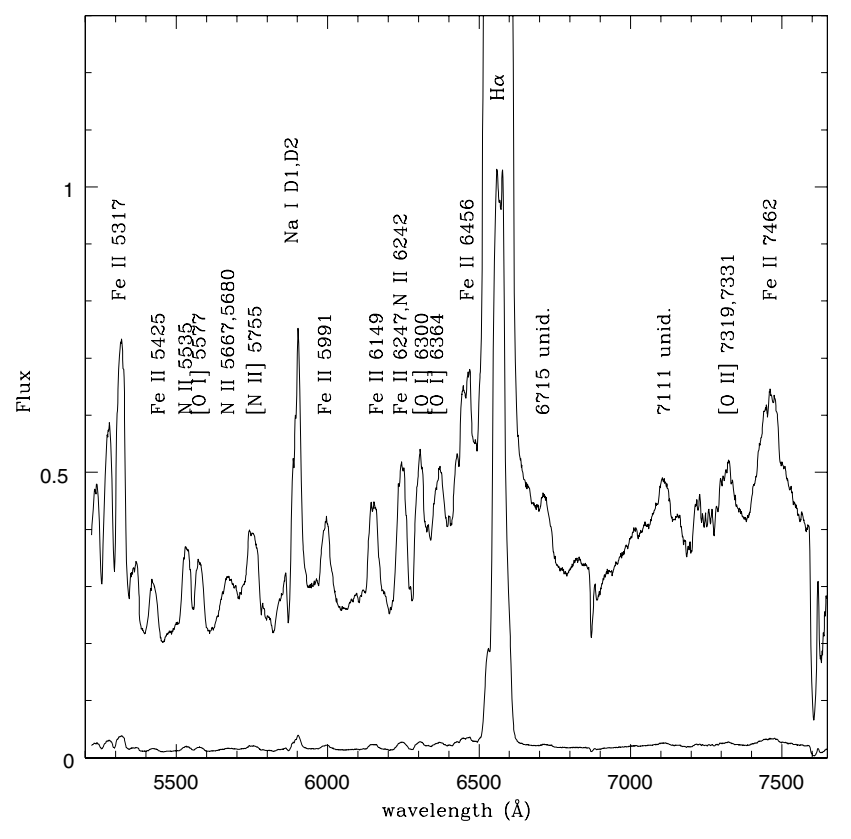

Fig. 11. A red spectrum of V2468 Cyg on 2008 March 23. The unit of the ordinate is $10^{-12} \mathrm{erg} \mathrm{cm}^{-2} \mathrm{~s}^{-1} \AA^{-1}$. The profile of $\mathrm{H} \alpha$ is shown by one twentieth scale.

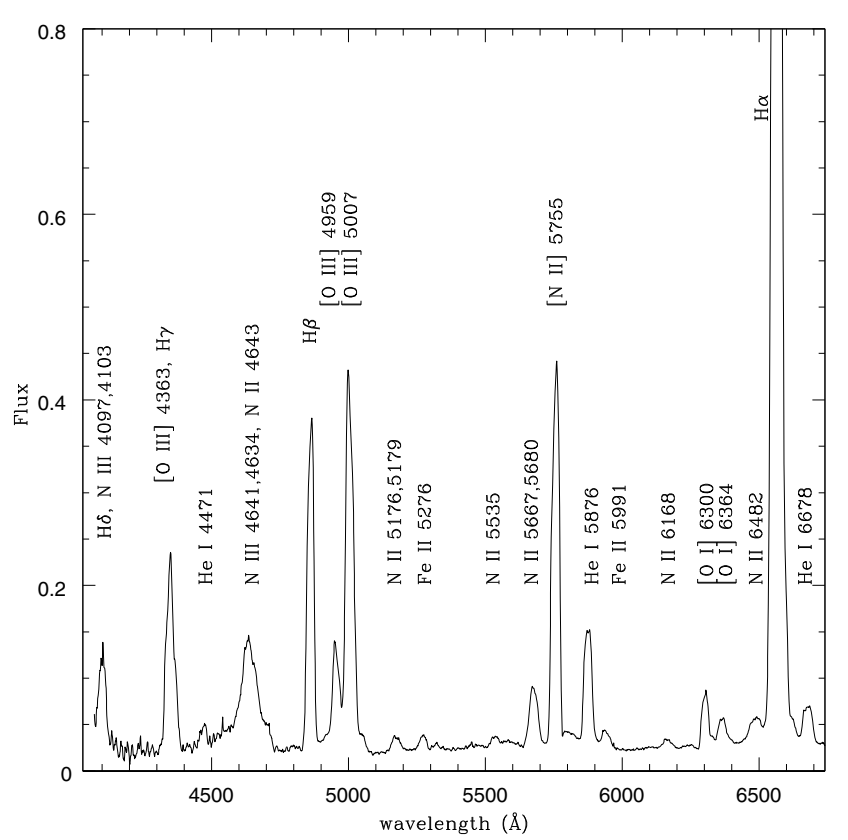

Fig. 12. A spectrum of V2468 Cyg on 2008 July 8. The unit of the ordinate is $10^{-12} \mathrm{erg} \mathrm{cm}^{-2} \mathrm{~s}^{-1} \AA^{-1}$.

(Sect. 6). The emission line [Fe VII] + [Ca V] 6087 was seen on October 9 (Fig. 14), while it was not detected on November 13 (Fig. 15 and Table 2). The emission lines of the other highly ionised ions, e.g., He II, [Fe VI], and [Ca V], also faded in November, while those of low ionised ions, e.g., He I and N II, strengthened (Table 2). A small brightening was seen during JD $2454770-80$ in November 2008 (Fig. 1). It seems that there was a temporary decrease in the temperature of the ionising source, which was probably due to its expansion. 
T. Iijima and H. Naito : Spectral evolution of the nova V2468 Cygni

Table 2. Intensities of prominent emission lines relative to $\mathrm{H} \beta=100$ of V2468 Cyg.

\begin{tabular}{|c|c|c|c|c|c|c|c|c|c|}
\hline \multirow[b]{2}{*}{$\lambda_{\mathrm{obs}}$} & \multirow[b]{2}{*}{ Identification } & \multicolumn{2}{|c|}{2008 Oct. 09} & \multicolumn{2}{|c|}{2008 Nov. 13} & \multicolumn{2}{|c|}{2009 June 3} & \multicolumn{2}{|c|}{2009 Sep. $27 / 28$} \\
\hline & & $F(\lambda)$ & $I(\lambda)$ & $F(\lambda)$ & $I(\lambda)$ & $F(\lambda)$ & $I(\lambda)$ & $F(\lambda)$ & $I(\lambda)$ \\
\hline 3428.6 & {$[\mathrm{Ne} \mathrm{V}] 3425.8$} & & & & & 120: & 303: & 210: & 528: \\
\hline 3586.3 & He I 3587.3, [Fe VII] 3587.2 & & & & & 11.5 & 25.6 & 19.4 & 43.4 \\
\hline 3725.8 & [O II] $3726.2,3728.9$ & & & & & 6.8 & 13.9 & 12.1 & 24.6 \\
\hline 3758.5 & {$[\mathrm{Fe}$ VII $] 3759.9$} & & & & & 27.2 & 54.4 & 38.1 & 76.1 \\
\hline 3812.3 & He II 3813.5 & & & & & 6.8: & 13.1: & 15.9 & 30.7 \\
\hline 3835.7: & H I 3835.4 & & & & & 9.7 & 18.5 & 12.4: & 23.5: \\
\hline 3869.3 & {$[\mathrm{Ne}$ III] 3868.7} & & & & & 68.4 & 127 & 61.2 & 114 \\
\hline 3892.6 & H I 3889.1, [Fe V] 3891.8,95.7 & & & & & 13.6: & 24.9: & 17.7 & 32.5 \\
\hline 3968.4 & $\mathrm{H} \epsilon,[\mathrm{Ne}$ III] 3967.5 & & & & & 30.0 & 52.8 & 26.7 & 47.0 \\
\hline 4073.0 & [Fe V] 4071.5, [S II] 4076.2 & & & & & 7.1 & 11.8 & 7.8 & 12.9 \\
\hline 4100.5 & $\mathrm{H} \delta, \mathrm{N}$ III $4103.9,4097.3$ & 37: & 61: & 46: & 76: & 38: & 63: & 40: & 66: \\
\hline 4196.9 & N III 4195.7 & $\ldots$ & $\ldots$ & $\ldots$ & $\ldots$ & 4.9 & 7.6 & 8.3 & 12.9 \\
\hline 4338.9 & $\mathrm{H} \gamma$ & 25: & 37: & 26: & 38: & 28: & 41: & 28: & 41: \\
\hline 4362.8 & [O III] 4363.0 & 128 & 184 & 121 & 173 & 171 & 246 & 148 & 213 \\
\hline 4473.5 & He I 4471.5, Mg II 4481.1 & 4.7 & 6.2 & 7.9 & 10.4 & 5.3 & 7.0 & 11.4 & 15.1 \\
\hline 4519.4: & N III 4518.2, 4523.6 & 9.7 & 12.3 & 5.1 & 6.5 & 18.8 & 23.9 & 29: & 37: \\
\hline 4606.9 & N II 4607.2 & 8.7 & 10.4 & 20.1 & 24.0 & 27.9 & 33.2 & 36.4 & 43.4 \\
\hline 4638.9 & N III 4640.6, 41.9, N II 4643.1 & 64.5 & 75.1 & 82.2 & 95.8 & 76.0 & 88.4 & 76.6 & 89.2 \\
\hline 4687.1 & He II 4685.7 & 31.9 & 36.0 & 23.0 & 25.9 & 71.2 & 80.1 & 84.4 & 95.0 \\
\hline 4721.2: & {$[\mathrm{Ne}$ IV] $4714.0,4720.0$} & 4.4: & 4.8: & 3.4: & 3.8: & 8.7 & 9.5: & 10.3: & 11.3: \\
\hline 4861.7 & $\mathrm{H} \beta$ & 100 & 100 & 100 & 100 & 100 & 100 & 100 & 100 \\
\hline 4933.5: & [O III] 4931.8, [Ca VII] 4938.6 & 3.0: & 2.9: & .. & $\ldots$ & 9.3: & 8.8: & 17.1: & 16.3: \\
\hline 4958.6 & [O III] 4958.9 & 127 & 119 & 154 & 144 & 348 & 327 & 388 & 365 \\
\hline 5006.9 & [O III $] 5006.8$ & 400 & 364 & 504 & 459 & 1027 & 937 & 1104 & 1007 \\
\hline 5046.8 & Si II 5041.1, 5056.0 & 8.9: & 7.9: & 4.7: & 4.2: & 7.5: & $6.7:$ & 8.7: & 7.8: \\
\hline 5148.4 & {$[\mathrm{Fe} \mathrm{VI}] 5146.8$} & 3.9 & 3.2 & ... & $\ldots$ & 11.4: & 9.6: & 19.4 & 16.3 \\
\hline 5174.9 & {$[\mathrm{Fe} \mathrm{VI}] 5177.0$} & 12.7 & 10.5 & 6.1 & 5.1 & 26.3 & 21.8 & 33.4 & 27.6 \\
\hline 5279.3 & {$[\mathrm{Fe} \mathrm{VI}] 5279.2,[\mathrm{Fe} \mathrm{VII}] 5276.1$} & 4.7 & 3.7 & 4.4 & 3.4 & 17.3 & 13.5 & 27.1 & 21.1 \\
\hline 5308.2 & {$[\mathrm{Ca} \mathrm{V}] 5308.9$} & 5.6 & 4.3 & .. & $\ldots$ & 23.1 & 17.7 & 27.7 & 21.3 \\
\hline 5416.6 & He II 5411.5 & 3.9 & 2.8 & 2.6 & 1.9 & 11.2 & 8.1 & 16.9 & 12.2 \\
\hline 5536.0 & N II 5535.4 & 3.0 & 2.0 & 4.1 & 2.8 & 2.4 & 1.6 & $\ldots$ & $\ldots$ \\
\hline 5617.2 & [Ca VII] 5615.8 & $\ldots$ & $\ldots$ & $\ldots$ & $\ldots$ & 10.4 & 6.8 & 17.4 & 11.3 \\
\hline 5676.9 & {$[\mathrm{Fe} \mathrm{VI}]$ 5678.0, N II 5679.6} & 20.2 & 12.8 & 22.1 & 14.0 & 15.4 & 9.8 & 19.1 & 12.1 \\
\hline 5721.8 & [Fe VII] 5720.9 & $\ldots$ & $\ldots$ & $\ldots$ & $\ldots$ & 36.3 & 22.5 & 80.5 & 50.0 \\
\hline 5754.1 & {$[\mathrm{~N}$ II] 5754.8} & 75.4 & 46.1 & 86.9 & 53.1 & & & 65.8 & 40.2 \\
\hline 5802.7 & C IV 5801.5? & $\ldots$ & $\ldots$ & $\ldots$ & $\ldots$ & & & 11.2: & 6.7: \\
\hline 5875.2 & He I 5875.6 & 33.1 & 19.1 & 38.5 & 22.3 & & & 25.7 & 14.9 \\
\hline 5938.4 & N II 5940.3, 5941.7 & 5.5 & 3.1 & 6.5 & 3.6 & & & 3.1: & 1.6: \\
\hline 6087.1 & [Fe VII] 6087.0, [Ca V] 6086.9 & 19.5 & 10.3 & $\ldots$ & $\ldots$ & & & 180 & 95 \\
\hline 6308.4 & [O I] 6300.2, [S III] 6310.2 & 12.1 & 5.9 & 8.5 & 4.1 & & & 10.9 & 5.3 \\
\hline 6369.9 & [O I] 6363.9, Si II 6371.4 & 13.0: & 6.2 : & 6.4: & 3.0: & & & 8.8 & 4.1 \\
\hline 6433.1: & {$[\mathrm{Ar} \mathrm{V}] 6434.9$} & $\ldots$ & $\ldots$ & $\ldots$ & $\ldots$ & & & 5.9: & 2.7: \\
\hline 6482.4: & N II 6482.1 & 4.1: & 1.9: & 5.2: & 2.3: & & & 10.0: & 4.5: \\
\hline 6563.4 & $\mathrm{H} \alpha$ & 840 & 369 & 847 & 372 & & & 804 & 354 \\
\hline 6593.3 & [N II] 6583.6, [Fe VII] 6599.1 & $\ldots$ & $\ldots$ & $\ldots$ & $\ldots$ & & & 13.0: & 5.6 \\
\hline 6678.4 & He I 6678.1 & 12.8 & 5.4 & 13.2 & 5.6 & & & 10.8 & 4.6 \\
\hline 6743.4 & C II $6750.2,6738.4$ & & & & & & & 7.8 & 3.2 \\
\hline 7006.1 & {$[\mathrm{Ar}$ V] 7006.3} & & & & & & & 25.0 & 9.5 \\
\hline 7064.3 & He I 7065.2 & & & & & & & 24.8 & 9.2 \\
\hline 7139.3 & [Ar III] 7135.8 & & & & & & & 35.1 & 12.8 \\
\hline 7325.5 & [O II] 7319.4, 7330.7 & & & & & & & 42.0 & 14.5 \\
\hline 7586 & He II $7592 ?$ & & & & & & & 18.1 & 5.8 \\
\hline 7715 & unid. & & & & & & & 48.4 & 15.0 \\
\hline $\mathrm{H} \beta$ & $10^{-12} \mathrm{erg} \mathrm{cm}^{-2} \mathrm{~s}^{-1}$ & 2.89 & 34.0 & 2.81 & 33.0 & 0.572 & 6.71 & 0.289 & 3.40 \\
\hline
\end{tabular}

Notes. $F(\lambda)$ : measured fluxes. $I(\lambda)$ : intensities corrected for $E(B-V)=0.77$.

Even in the medium dispersion spectra, it is possible to see the variations in the profiles of some emission lines. For example, the peaks of the emission lines of [O III] were blueshifted with respect to the centre of the lines on July 8 and 24
(Figs. 12, 13), while they were red-shifted on October 9 (Fig. 14) and November 13 (Fig. 15). The variable profiles of the emission lines at the nebular stage were notable peculiarities of this nova. 


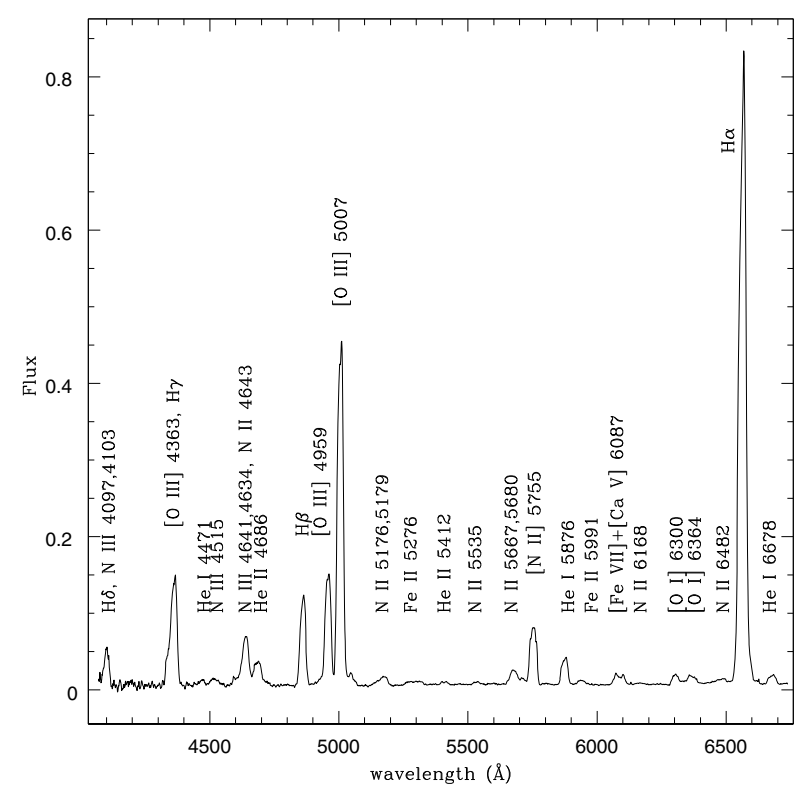

Fig. 14. A spectrum of V2468 Cyg on 2008 October 9. The unit of the ordinate is $10^{-12} \mathrm{erg} \mathrm{cm}^{-2} \mathrm{~s}^{-1} \AA^{-1}$.

The variations in the profiles were more clearly observed in 2009 as reported in Sect. 5.2.

Lynch et al. (2008) reported that they found infrared coronal emission lines [Si VI], [S VIII], [S IX], [Al IX], and [Ca VIII] in the spectra obtained on 2008 October 4 by the Infrared Telescope Facility. In the optical region, however, we did not find any coronal line, e.g., $[\mathrm{Fe} \mathrm{X}] 6375$, in the spectra obtained in October 2008 and later.

\subsection{Spectral evolution in 2009}

Following the normal spectral evolution of classical novae, the emission lines of [O III], [N II], [Ne III] etc. strengthened in 2009. The emission line of $[\mathrm{Fe} \mathrm{VII}]+[\mathrm{Ca} \mathrm{V}] 6087$ was the strongest in the red region after $\mathrm{H} \alpha$. Schwarz et al. (2009) reported that the prominent emission lines in the spectrum obtained on 2009 July 12 were H I Balmer lines and the forbidden lines of [Ne III], [O III], [N II], and [Fe VII], which was consistent with our results.

Figure 16 shows a tracing of the spectrum obtained on 2009 June 3, where the emission line [O III] 5007 was roughly ten times stronger than $\mathrm{H} \beta$ and the emission line of [Ne V] 3426 was prominent in the blue region. Intensities of selected emission lines in this spectrum were measured (Sect. 6).

Figure 17 shows a tracing of the spectrum obtained on 2009 July 21. A peculiar phenomenon in this spectrum was the very broad emission feature around $3810 \AA$. This phenomenon is discussed in detail in Sect. 7.2. The other parts of the spectrum were nearly the same as those of June 3 (Fig. 16).

Figure 18 shows a tracing of the red spectrum obtained on 2009 September 27, and Fig. 19 shows a tracing of the blue spectrum obtained on September 28. Intensities of selected emission lines in these spectra were measured (Sect. 6).

Figure 20 shows a tracing of the spectrum obtained on 2009 October 15. The profiles of some emission lines had changed significantly with respect to those in September (Fig. 19). This phenomenon is reported in detail in Sect. 5.2.

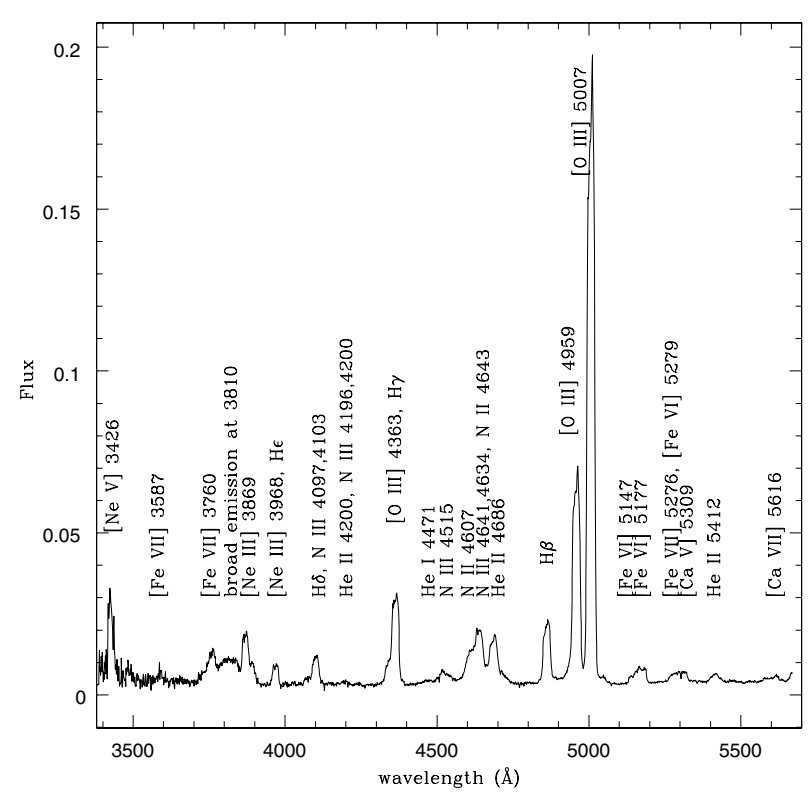

Fig. 17. A spectrum of V2468 Cyg on 2009 July 21. The unit of the ordinate is $10^{-12} \mathrm{erg} \mathrm{cm}^{-2} \mathrm{~s}^{-1} \AA^{-1}$.

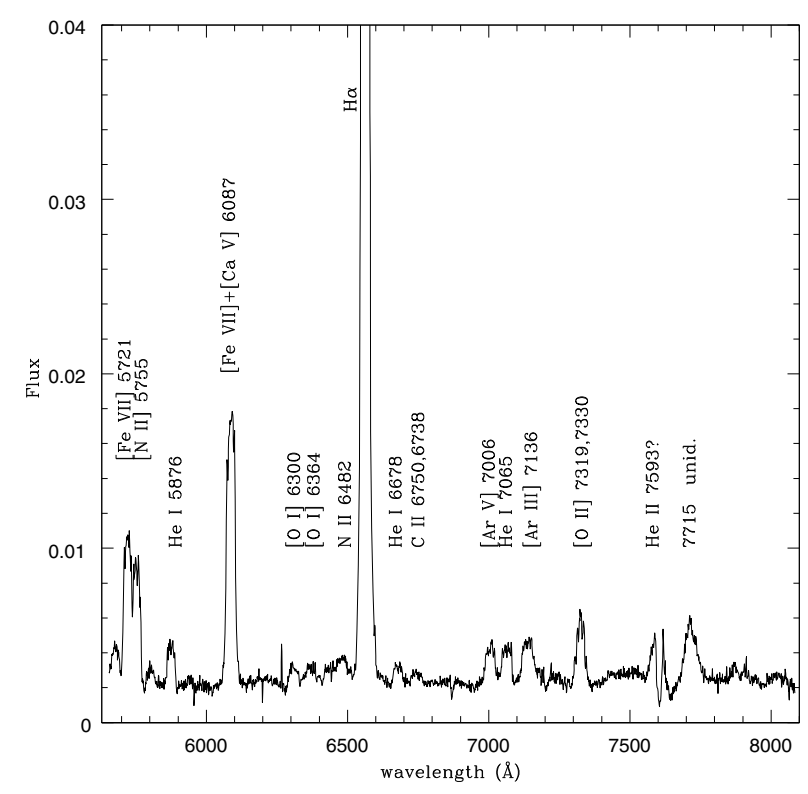

Fig. 18. A spectrum of V2468 Cyg on 2009 September 27. The unit of the ordinate is $10^{-12} \mathrm{erg} \mathrm{cm}^{-2} \mathrm{~s}^{-1} \AA^{-1}$.

\section{Profiles of the emission lines}

\subsection{Early decline stage}

Figures 21-24 show the profiles of $\mathrm{H} \alpha, \mathrm{H} \beta, \mathrm{H} \gamma$, and Fe II 5018 in the early decline stage. The ordinates are arbitrary intensity scales and the height of each tracing is normalised at unity. The abscisse are the heliocentric radial velocities in $\mathrm{km} \mathrm{s}^{-1}$. The positions of the heliocentric radial velocity $+660 \mathrm{~km} \mathrm{~s}^{-1}$ are indicated by broken lines.

All lines showed simple P Cygni type profiles on 2008 March 8. The radial velocities of the absorption components of most lines were found in the range $-790 \pm 80 \mathrm{~km} \mathrm{~s}^{-1}$. The absorption components weakened with time and their blue-shifts 


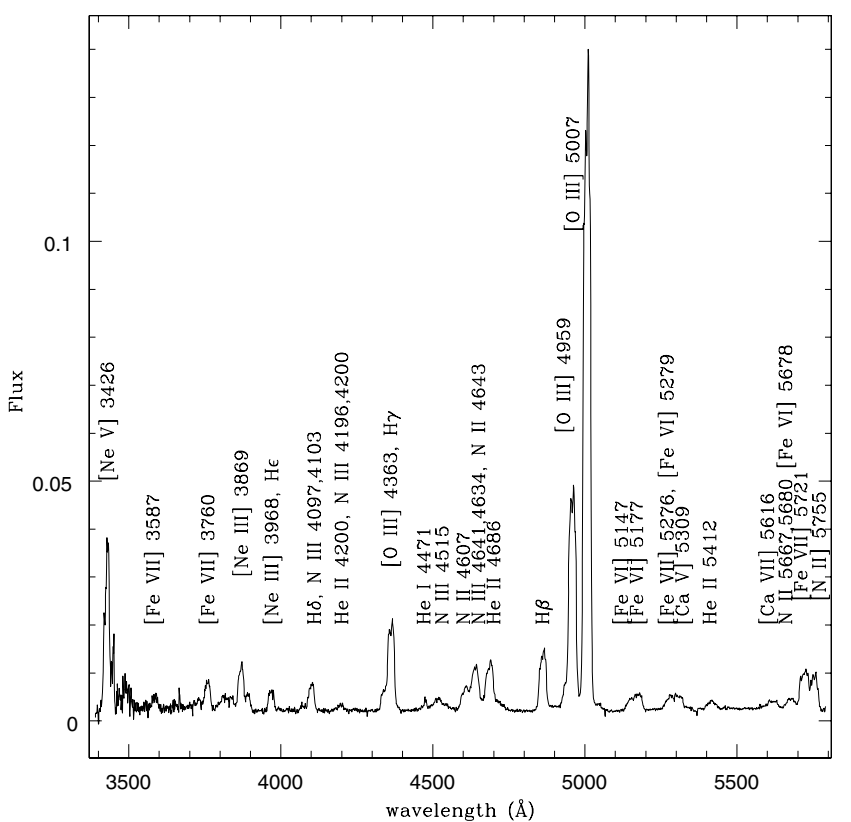

Fig. 19. A spectrum of V2468 Cyg on 2009 September 28. The unit of the ordinate is $10^{-12} \mathrm{erg} \mathrm{cm}^{-2} \mathrm{~s}^{-1} \AA^{-1}$.

increased. One strange exception was the case of $\mathrm{H} \alpha$ whose minimum blue-shift was observed on March 10 (Fig. 21). The radial velocities of the absorption component of $\mathrm{H} \alpha$ were $-910 \mathrm{~km} \mathrm{~s}^{-1}$ on March 8, $-850 \mathrm{~km} \mathrm{~s}^{-1}$ on March 10 , and $-910 \mathrm{~km} \mathrm{~s}^{-1}$ on March 11 with errors of $\pm 20 \mathrm{~km} \mathrm{~s}^{-1}$. The red-ward side of the absorption component may have been filled by the emission component on March 8. In the period from March 12 to 15, the mean radial velocity of the first absorption components of $\mathrm{H} \mathrm{I}$ and Fe II lines was about $-1050 \pm 20 \mathrm{~km} \mathrm{~s}^{-1}$, and new absorption components with velocities of $-1700 \pm 20 \mathrm{~km} \mathrm{~s}^{-1}$ appeared. The mean radial velocities of these absorption components were $-1180 \pm 20 \mathrm{~km} \mathrm{~s}^{-1}$ and $-2020 \pm 20 \mathrm{~km} \mathrm{~s}^{-1}$ on March 18 .

Emission peaks with radial velocities of $+660 \pm 30 \mathrm{~km} \mathrm{~s}^{-1}$ were seen on some tracings. That of $\mathrm{H} \beta$ was seen on March 10 and 11, but was not seen or very weak on March 12 and 13 (Fig. 22). It appeared again on March 15 and weakened on March 18, then strengthened on March 23. The variation in the emission peak of Fe II 5018 was nearly the same as that of $\mathrm{H} \beta$, except for its weakness on March 23 (Fig. 24). The $\mathrm{H} \alpha$ emission line did not peak at $+660 \mathrm{~km} \mathrm{~s}^{-1}$ on March 10 (Fig. 21) in contrast to the profile of $\mathrm{H} \beta$, but peaked instead on March 15 and 23. The red-ward parts were always dominant in $\mathrm{H} \gamma$ and the higher members of the Balmer series (Fig. 23 for $\mathrm{H} \gamma$ ). The Balmer decline rate appears to have been steeper in the blue-ward parts of the emission lines than in the red-ward parts. The Balmer decline rate largely varies according to the self-absorption of the H I lines (Netzer 1975). The physical conditions in the emitting region of the red-ward parts of the lines, the electron temperature, and/or electron density, may have been different from that in the emitting region of the blue-ward parts.

It seems that the emission peaks at $+660 \mathrm{~km} \mathrm{~s}^{-1}$ weakened when the absorption components became deep. However, we do not know why the emission peaks repeatedly appeared and disappeared. If a mass of gas had been ejected with the radial velocity $+660 \mathrm{~km} \mathrm{~s}^{-1}$, the corresponding emission peaks should have been observed continually after the ejection.

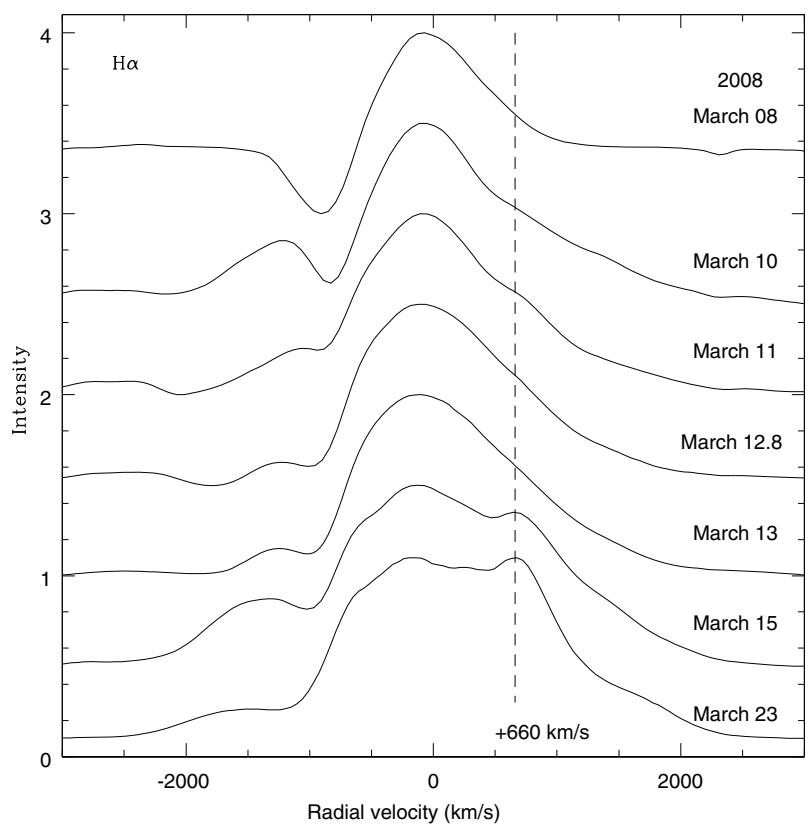

Fig. 21. Profiles of $\mathrm{H} \alpha$ in the early decline stage. The position of the heliocentric radial velocity $+660 \mathrm{~km} \mathrm{~s}^{-1}$ is indicated by a broken line.

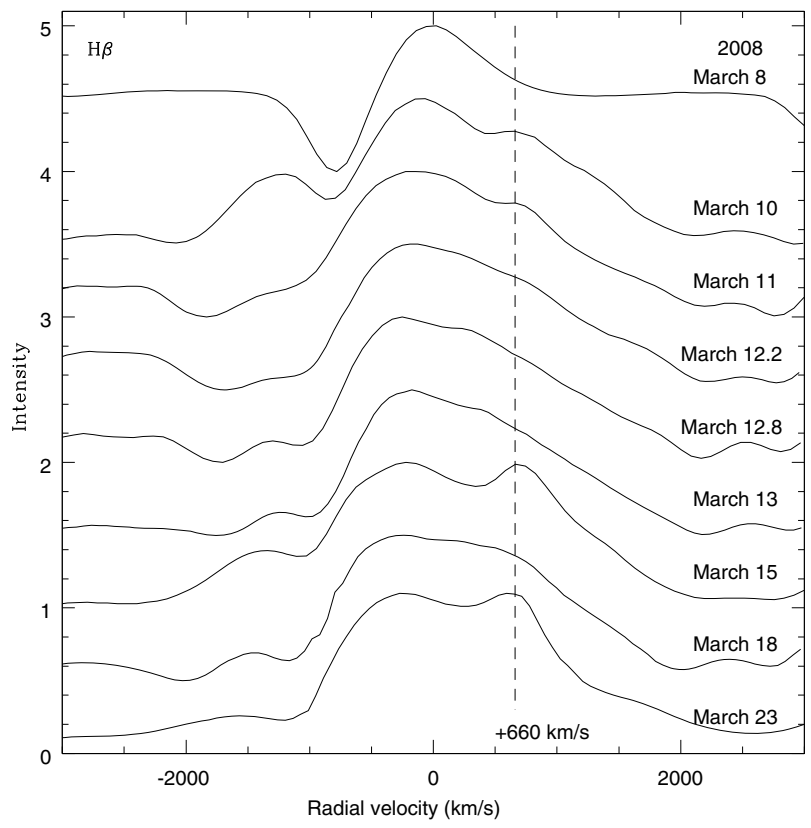

Fig. 22. Profiles of $\mathrm{H} \beta$ in the early decline stage. The position of the heliocentric radial velocity $+660 \mathrm{~km} \mathrm{~s}^{-1}$ is indicated by a broken line.

\subsection{Profiles of emission lines in 2009}

Profiles of emission lines of usual classical novae do not vary in the nebular stage. This is because the mass ejection terminates and the structure of the ejected shell does not change except for the simple expansion. However, the profiles of some emission lines in the spectra of V2468 Cyg varied significantly during the nebular stage, even when more than one year passed from light maximum. 


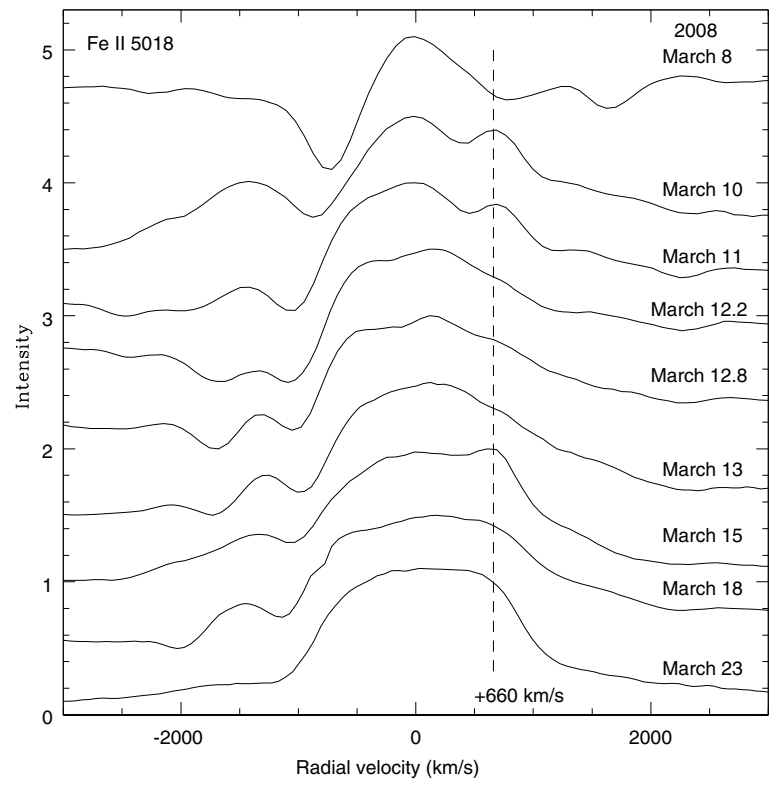

Fig. 24. Profiles of Fe II 5018 in the early decline stage. The position of the heliocentric radial velocity $+660 \mathrm{~km} \mathrm{~s}^{-1}$ is indicated by a broken line.

The profiles of $\mathrm{H} \alpha, \mathrm{H} \beta$, [O III] 5007, He II 4686, [Fe VII] 5721, and [Fe VII] + [Ca V] 6087 in 2009 are shown in Figs. 25-30. Profiles of $\mathrm{H} \alpha$ on 2008 October 9 and November 13 are added in Fig. 25, and some profiles of [Fe VII] + [Ca V] 6087 in 2008 are added in Fig. 30. The abscisse are heliocentric radial velocities in $\mathrm{km} \mathrm{s}^{-1}$, and the ordinates are arbitrary intensity scales. The height of each tracing is normalised at unity. The rather smooth profiles of $\mathrm{H} \beta$ and [O III] 5007 on June 3 were due to a technical problem in the spectrograph. As seen in the figures, the emission lines of $\mathrm{H} \alpha, \mathrm{H} \beta$, and [O III] 5007 had four main peaks, whose mean radial velocities were $-640 \mathrm{~km} \mathrm{~s}^{-1},-260 \mathrm{~km} \mathrm{~s}^{-1},+255 \mathrm{~km} \mathrm{~s}^{-1}$, and $+620 \mathrm{~km} \mathrm{~s}^{-1}$ with errors of $\pm 10 \mathrm{~km} \mathrm{~s}^{-1}$. The positions of these radial velocities are indicated by broken lines in the figures. The profiles of $\mathrm{H} \alpha$ and $\mathrm{H} \beta$ varied widely during the early decline stage (Sect. 5.1), but no significant variation was detected in 2009 (Figs. 25, 26). It seems that the variation in the profile of $\mathrm{H} \alpha$ terminated on October 2008 (Fig. 25), because the profiles at that time and on November 13 did not differ significantly from those in 2009. The smooth profiles of $\mathrm{H} \alpha$ in 2008 were caused by to the lower resolution of the spectrograph.

The emission lines of $\mathrm{H} \beta$, [O III] 5007, and He II 4686 displayed similar profiles on 2009 July 21 . The reddest peak of He II 4686 strengthened slightly on September 28 (Fig. 28), while the profile of [O III] 5007 did not change (Fig. 27). Drastic variations in the profiles were observed on October 15, which was 587 days after light maximum. In particular, the reddest peak of [O III] 5007 strengthened and that of He II 4686 faded, while the profile of $\mathrm{H} \beta$ hardly changed (Figs. 26-28). When the next spectrum was obtained on October 26 , these three lines delineated profiles those were nearly symmetric and similar each other (Figs. 26-28).

As seen in Figs. 29 and 30, the profiles of [Fe VII] 5721 and $[\mathrm{Fe} \mathrm{VII}]+[\mathrm{Ca} \mathrm{V}] 6087$ were more complicated and their variations were more drastic than those of the [O III] and He II lines. The reddest peak of [Fe VII] 5721 also faded on October 15, as did the He II 4686 line, while its profile was fairly different from

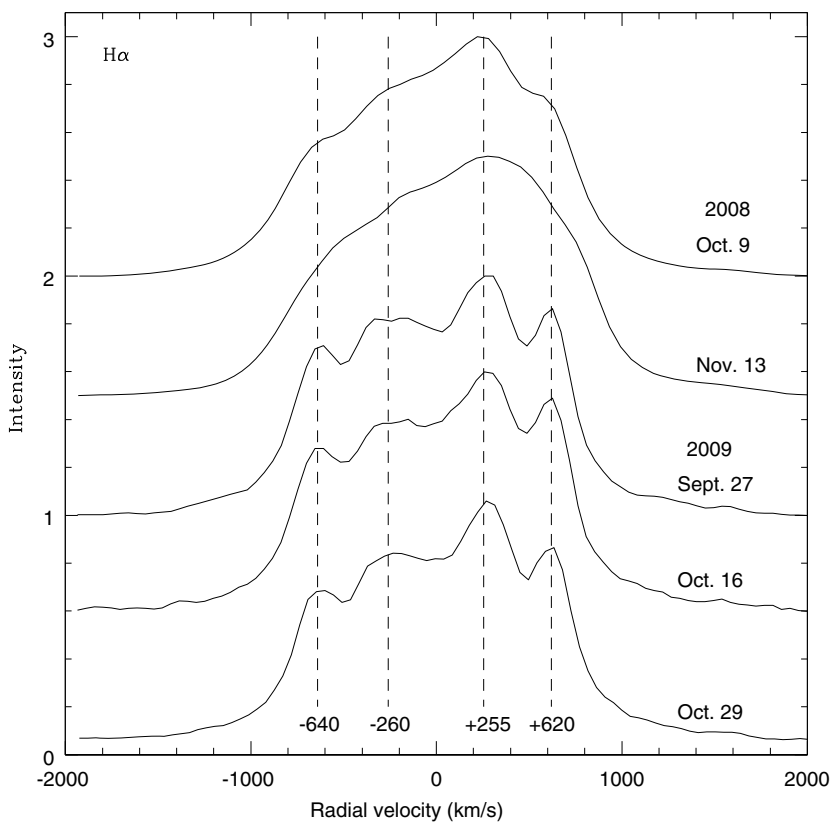

Fig. 25. Profiles of $\mathrm{H} \alpha$ in 2008 and 2009. The positions of the heliocentric radial velocities $-640,-260,+255$, and $+620 \mathrm{~km} \mathrm{~s}^{-1}$ are indicated by broken lines.

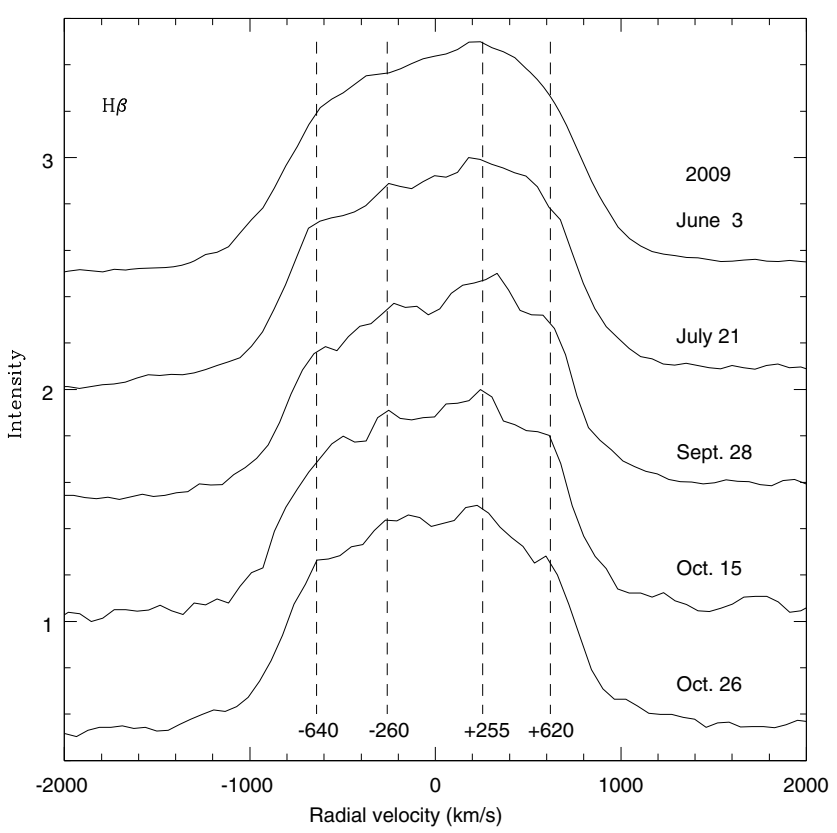

Fig. 26. Profiles of $\mathrm{H} \beta$ in 2009. The positions of the heliocentric radial velocities $-640,-260,+255$, and $+620 \mathrm{~km} \mathrm{~s}^{-1}$ are indicated by broken lines.

that of He II 4686 on October 26 (Fig. 29). The emission lines of [Fe VII] 5721 and [Fe VII] + [Ca V] 6087 showed convex profiles in September similar to those of the other lines, while only these lines showed concave profiles on October 26 and 29 (Figs. 29, 30).

The profile of [Fe VII] + [Ca V] 6087 also varied widely in 2008 (Fig. 30). The radial velocity of the line centre was $-350 \pm$ $20 \mathrm{~km} \mathrm{~s}^{-1}$ on July 24 and $+230 \pm 20 \mathrm{~km} \mathrm{~s}^{-1}$ on August 9, i.e., the 


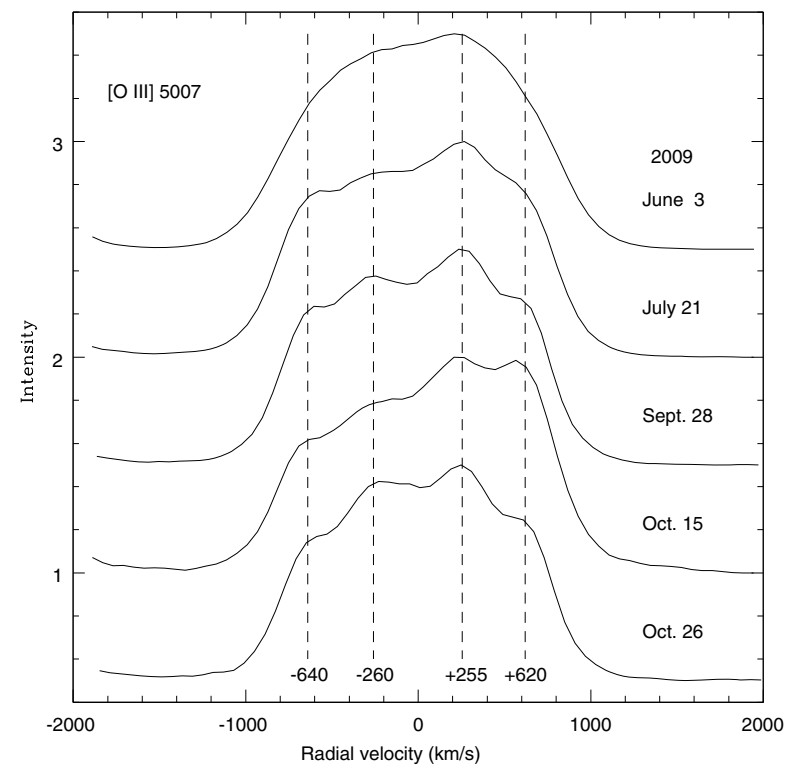

Fig. 27. Profiles of [O III] 5007 in 2009. The positions of the heliocentric radial velocities $-640,-260,+255$, and $+620 \mathrm{~km} \mathrm{~s}^{-1}$ are indicated by broken lines.

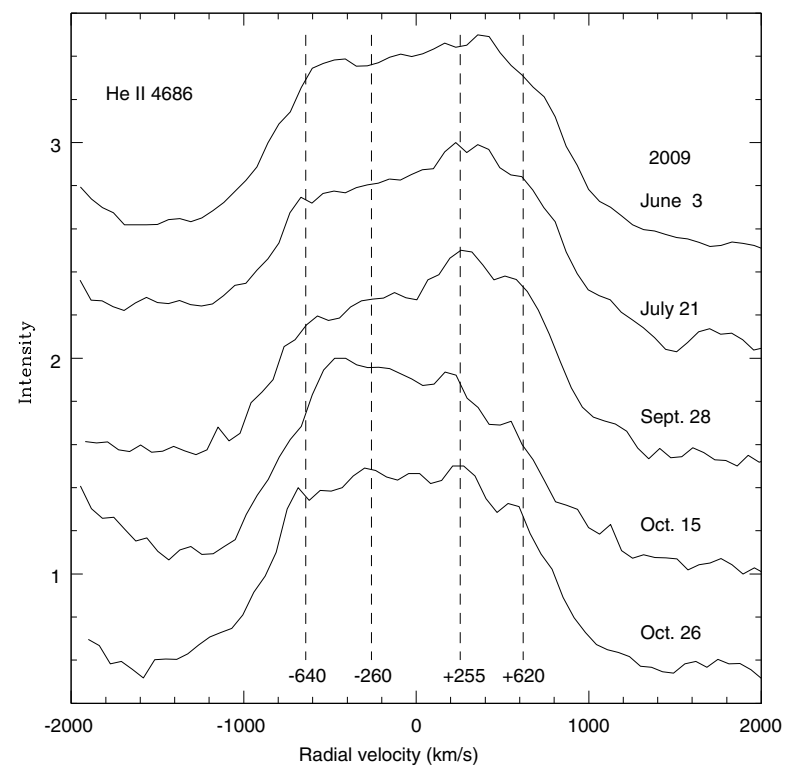

Fig. 28. Profiles of He II 4686 in 2009. The positions of the heliocentric radial velocities $-640,-260,+255$, and $+620 \mathrm{~km} \mathrm{~s}^{-1}$ are indicated by broken lines.

line centre had shifted $580 \mathrm{~km} \mathrm{~s}^{-1}$ in 16 days. The shifts of the line centre of the other lines in the same period were $200 \mathrm{~km} \mathrm{~s}^{-1}$ for He II 4686, $78 \mathrm{~km} \mathrm{~s}^{-1}$ for both [O III] 5007 and [N II] 5755 , $65 \mathrm{~km} \mathrm{~s}^{-1}$ for He I 5876, and $38 \mathrm{~km} \mathrm{~s}^{-1}$ for $\mathrm{H} \beta$, where the errors were about $\pm 20 \mathrm{~km} \mathrm{~s}^{-1}$. The radial velocity of the line centre of $[\mathrm{Fe} \mathrm{VII}]+[\mathrm{Ca} \mathrm{V}] 6087$ on 2008 October 9 was $-80 \mathrm{~km} \mathrm{~s}^{-1}$, and there were two peaks at $-660 \mathrm{~km} \mathrm{~s}^{-1}$ and $+680 \mathrm{~km} \mathrm{~s}^{-1}$ (Fig. 30). No other line in the same spectrum had such a broad double peak profile (Fig. 14 and Fig. 25 for $\mathrm{H} \alpha$ ).

It seems that the variable emission peaks at $+660 \mathrm{~km} \mathrm{~s}^{-1}$ of $\mathrm{H}$ I lines in the early decline stage and those at $+620 \mathrm{~km} \mathrm{~s}^{-1}$ of

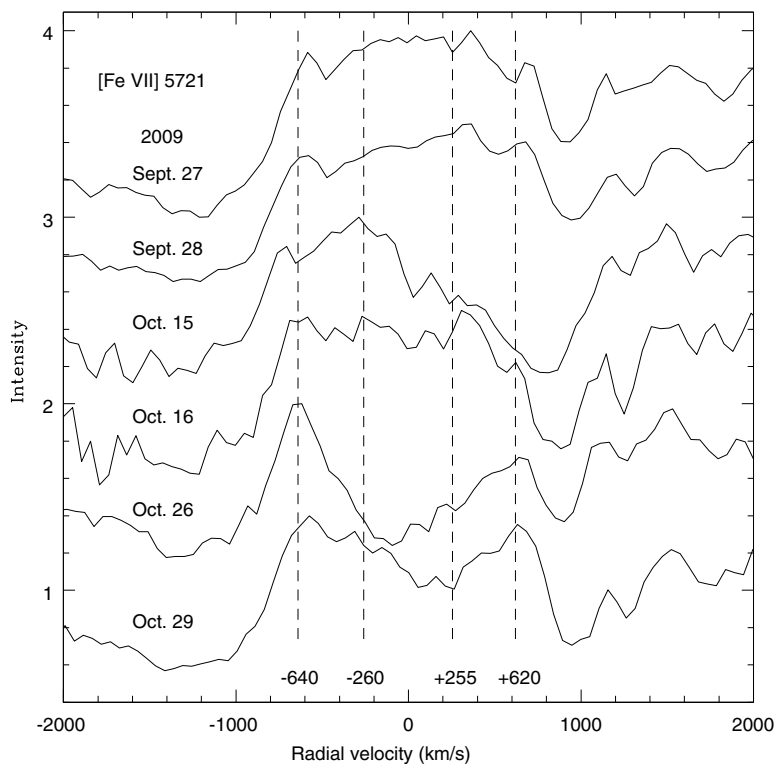

Fig. 29. Profiles of [Fe VII] 5721 in 2009. The positions of the heliocentric radial velocities $-640,-260,+255$, and $+620 \mathrm{~km} \mathrm{~s}^{-1}$ are indicated by broken lines.

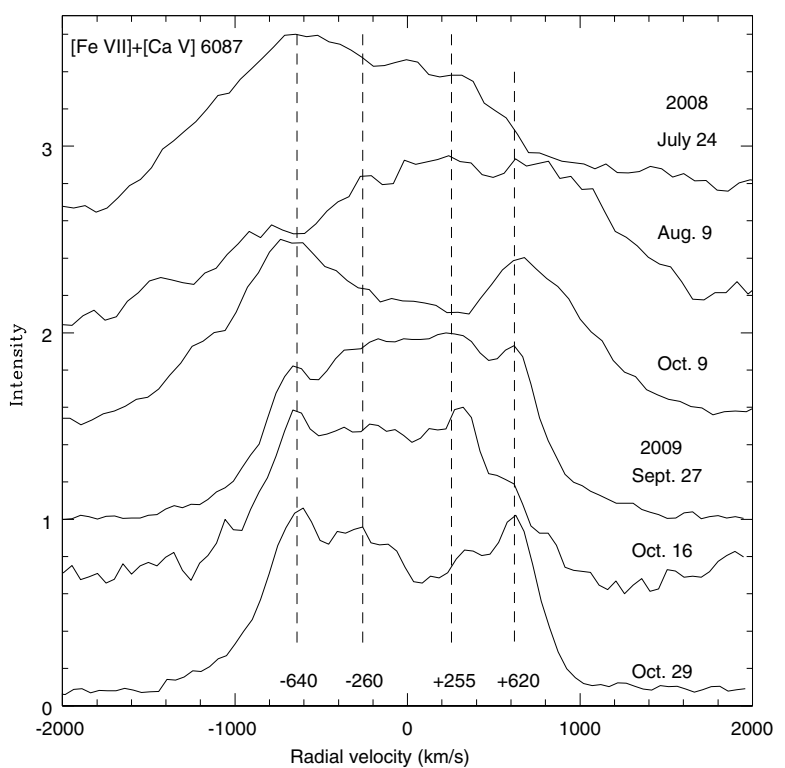

Fig. 30. Profiles of [Fe VII] $+[\mathrm{Ca} \mathrm{V}] 6087$ in 2008 and 2009. The positions of the heliocentric radial velocities $-640,-260,+255$, and $+620 \mathrm{~km} \mathrm{~s}^{-1}$ are indicated by broken lines.

both [O III] and He II in 2009 probably corresponded to the same mass of gas. The difference in radial velocity could have been due to an interaction between the mass of gas and the interstellar matter. However, the cause of the variability in intensity of the emission peaks is not known. The lack of variability in the $\mathrm{H}$ I line profiles in 2009, in contrast to the variations in the other lines, is a mysterious phenomenon. If a new mass ejection had occurred, the profiles of the H I lines should have been the first to change.

The profiles of [Fe VII] 5721 and [Fe VII] + [Ca V] 6087 on 2009 September 27 and 28 were similar to that of the emission 
line from a model of nova shell with equatorial rings and polar caps at an inclination angle of 90 degrees (Fig. 3 of Gill \& O'Brien 1999). On the other hand, their profiles on October 26 and 29 were similar to that from the same nova shell, but at an inclination angle of 30 degrees (Gill \& O'Brien 1999). The inclination of the ejected shell of a nova indeed did not change during the nebular stage. A possible cause of the variations in the emission-line profiles was a change in the field of the ionising UV radiation of the white dwarf. It will be necessary to study the variations in the profiles of emission lines from a nova shell as the UV radiation field changes in a similar way to a search light because of, for example, the precession of the thick accretion disk. The accretion disk was probably in the course of being reconstructed when our observations in 2009 were carried out, because Schwarz et al. (2009) detected large amplitude variations in the $\mathrm{X}$-ray and $U V$ light curves during June and August 2009 on time-scales of about $500 \mathrm{~s}$ for X-rays.

\section{Intensities of emission lines}

The intensities of selected emission lines were measured in the spectra obtained on 2008 October 9, November 13, and 2009 June 3, and September 27 and 28. The intensities relative to $\mathrm{H} \beta=100$ are given in Table 2, where $F(\lambda)$ are the measured fluxes and $I(\lambda)$ are the intensities corrected for the interstellar extinction at $E(B-V)=0.77$ (Rudy et al. 2008). The absolute intensities of $\mathrm{H} \beta$ are given in the bottom line of Table 2 in units of $10^{-12} \mathrm{erg} \mathrm{cm}^{-2} \mathrm{~s}^{-1}$. The errors in the intensities are about $10 \%$, and the values of lower accuracy are denoted by a colon. Three dots in the table mean that the corresponding line was not detected, i.e., its intensity was roughly less than $1 \%$ of $\mathrm{H} \beta$. The red spectrum obtained on 2009 September 27 and the blue spectrum on September 28 were combined, because the spectral evolution was slow enough at that time.

We estimated some properties of the ejecta using the intensities of some emission lines of 2009 September 27 and 28. The intensity ratio of the nebular lines to the auroral line of [O III] was

$[I(5007)+I(4959)] / I(4363)=6.44$.

Using the formula of Seaton (1975), we estimated the electron density of the ejecta to be $6.2 \times 10^{6} \mathrm{~cm}^{-3}$, where we assumed the electron temperature at $10000 \mathrm{~K}$ to be a reasonable default one. The differences of $\pm 1000 \mathrm{~K}$ in the electron temperature correspond to differences of \pm 0.2 in the electron density on a logarithmic scale.

We derived an abundance of neutral helium relative to hydrogen in number as $N\left(\mathrm{He}^{0}\right)=0.11$ from the intensities of He I 5876 and He I 6678, where the detailed process is explained by Iijima (2006). The effects of the collisional excitation of the He I lines were corrected using the formulae of Peimbert \& Torres-Peimbert (1987). The emission line of He I 4471 was not used in our estimate, because it was blended with $\mathrm{Mg}$ II 4481. We derived $N\left(\mathrm{He}^{+}\right)=0.09$ from the intensity of the emission line He II 4686. Thus the total helium abundance is $N(\mathrm{He})=$ $0.20 \pm 0.01$.

The mass of the ejecta was estimated to be $1.7 \pm 1 \times 10^{-5} M_{\odot}$ from the intensities of H I, He I, and He II lines using the formulae given by Iijima (2006) with the distance $5.5 \mathrm{kpc}$. This result should be a lower limit to the mass of the ejecta, because only the mass of gas related to the emission lines was taken into account. If we use the intensities of the emission lines observed in 2008 (Table 2), we infer masses of the ejecta roughly one order of magnitude higher. However, we did not adopt those results, because a very high electron density was expected for the nebulosity and it was unclear whether the case B recombination process (Osterbrock 1989) was applicable.

\section{Unidentified emission features}

\subsection{Emission line at $7715 \AA$ and some others}

Figure 31 shows the red part of the spectrum obtained on 2009 September 27: the red part of Fig. 18. As seen in Figs. 18 and 31, there was a prominent emission line at $7715 \pm 0.5 \AA$. Its width $(F W H M)$ was $1750 \mathrm{~km} \mathrm{~s}^{-1}$, which was larger than those of the other lines in the same spectrum, namely $1390 \mathrm{~km} \mathrm{~s}^{-1}$ of $\mathrm{H} \alpha$ and $1480 \mathrm{~km} \mathrm{~s}^{-1}$ of $[\mathrm{Fe} \mathrm{VII}]+[\mathrm{Ca} \mathrm{V}] 6087$, He I 7065, and [Ar V] 7006. Its wider profile suggested that there were at least two components in the emission: a major component at about $7710 \AA$ and a minor component at 7720-7730 ̊.

We examined the spectra of V1016 Cyg, V1329 Cyg, and HM Sge in our archives. The spectra of these symbiotic stars are similar to those of classical novae in the nebular stage. We found three emission lines at 7711.5, 7716.8, and 7727.3 $\AA$ with errors of $\pm 0.7 \AA$ in the spectra of these objects. It seems that these emission lines depend on different elements, because the intensity ratios among them vary in the three objects.

The emission at $7715 \AA$ of V2468 Cyg possibly consisted of these lines. The minor component at 7720-7730 $\AA$ could have corresponded to the line at $7727.3 \AA$. We identified this line as C IV 7726.2 of unknown multiplet number (Meinel et al. 1968; NIST database ${ }^{2}$ ), because the emission line of C IV 5801.5 was probably detected in the same spectrum (Table 2). On the other hand, it is difficult to identify the other lines. An emission line at $7711 \pm 2 \AA$ was detected in the spectra of RS Oph during the outburst in 2006 (Iijima 2009). Its intensities were roughly $3 \%$ of $\mathrm{H} \beta$ when the coronal emission lines were prominent. Iijima (2009) was unable to identify this line, but suggested that it belonged to a coronal line. In the present case, however, it is unlikely that the line at $7711.5 \AA$ belongs to a coronal line, because no coronal line was detected in the spectra of either V2468 Cyg or the symbiotic stars. It is also unlikely that this line was due to Fe II 73, 7711.7 (Moore 1959), because no other Fe II line was detected in the same spectrum (Table 2). One candidate for the line at $7711.5 \AA$ is Si III 7710.7 (NIST database). However, we were unable to confirm this identification, because the other lines of Si III of the same multiplet, e.g., at $7844.2 \AA$ (NIST database), were not detected. The line at $7716.8 \AA$ also remains unidentified.

A prominent emission line was detected at about $7600 \AA$ (Figs. 18, 31). Part of this emission line may have been due to He II 6, 7592.7 (Meinel et al. 1968; Moore 1959), but it was far too incredibly intense with respect to the line of the same multiplet He II 7177.5 (Moore 1959) which was barely detected in the same spectrum (Figs. 18, 31). Unfortunately, the precise wavelength of this line was not measured, because of blending with an atmospheric absorption band.

Some weak emission features were detected at $6715 \AA$ and $7111 \AA$ in the early decline stage (Fig. 11). It is obvious that the line at $6715 \AA$ was not due to [S II] 6717, because the other line [S II] 6731 was not detected. The line at $7111 \AA$ was probably the same as the unidentified line at $7105 \pm 2.5 \AA$ detected in the spectra of the nova V1494 Aql (Iijima \& Esenoglu 2003).

${ }^{2}$ http://physics.nist.gov/cgi-bin/AtData/lines-form 


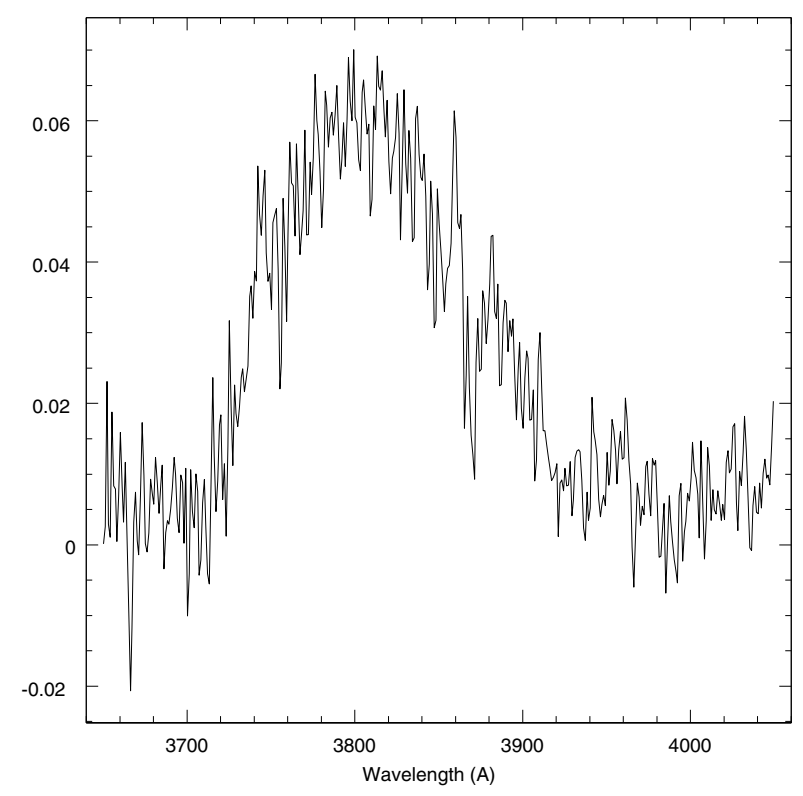

Fig. 32. The difference of the spectrum of 2009 July 21 from the average of the spectra of June 3 and September 28.

An identification as [Ni II] 13F, 7103 was suggested in the article, but was not confirmed, because no other line of [Ni II] was detected in the same spectrum (Iijima \& Esenoglu 2003). The identification of [Ni II] 7103 is not acceptable in the present case, because the difference in wavelength is much larger than the observational error.

\subsection{Broad emission feature around $3810 \AA$}

As seen in Fig. 17, a very broad emission feature appeared in the spectral range from $3790 \AA$ to $3850 \AA$ on 2009 July 21,500 days after light maximum. This emission feature was not seen in the earlier (e.g. Fig. 16) or later spectra (e.g. Fig. 19). We carefully checked the raw CCD image of the spectrum on which, however, we did not find any evidence that the emission feature was due to an artificial effect, i.e., scattering lights, a ghost, or something else. Some other objects were observed in the same night with exactly the same setting of the spectrograph. These included the rich emission-line objects BF Cyg and PU Vul, whose spectra are similar to that of V2468 Cyg. None of them, however, displayed such a broad emission feature. Thus, we have concluded that the broad emission feature around $3810 \AA$ was a real astronomical phenomenon in V2468 Cyg.

There were emission lines of He II 3813.5 and H I 3835.4 in the spectra of this nova in June and September 2009 (Table 2). However, these lines may not have contributed to the broad emission feature on July 21, because the other lines of H I and He II did not vary in intensity.

We took an average of the spectra of June 3 and September 28, which showed similar features in this spectral region (Figs. 16, 19), then subtracted it from the spectrum of July 21. The result is shown in Fig. 32, where the average spectrum was multiplied by an arbitrary factor to ensure that the continuum level of the difference corresponded to nearly zero.

The central wavelength of the broad emission feature was about $3808 \AA$ and the width $(F W H M)$ was $130 \AA$. The emission feature seems to have been caused by multiple lines of the same element, because it is extremely broad. The candidates for identification are the multiple lines of [Fe V] 3F, 3839.3, 3794.9, 3851.3, 3819.9 etc. and [Fe VI] 3F, 3847.4, 3883.2, and 3813.5 (Moore 1959; NIST database). We propose that the lines of $[\mathrm{Fe} \mathrm{V}]$ were more probable, because the emission lines of [Fe VI] at 5147 and $5177 \AA$ did not strengthen significantly on July 21 (Figs. 16, 17, and 19).

There might have been a resonance excitation of the $[\mathrm{Fe} \mathrm{V}]$ lines. At the present time, however, we have no definite model to explain this phenomenon. Further work, which is beyond the scope of this paper, is needed.

Acknowledgements. We are grateful to Prof.s R. Barbon, I. Hachisu, and M. Kato for the careful reading of the manuscript and useful suggestions. Thanks are also due to Mr. S. Narusawa for technical support during the observations at Nishi-Harima Observatory, and to Mr. S. Kiyota and the other members of VSNET Japan for the photometric data of V2468 Cyg. T.I. is grateful to Prof. T. Kuroda and the other staff members of Nishi-Harima Astronomical Observatory for friendly hospitality.

\section{References}

Beaky, M. M. 2008, IAU Circ., 8928

Cohen, J. G. 1975, ApJ, 197, 117

Della Valle, M., \& Livio, M. 1995, ApJ, 452, 704

Gill, C. D., \& O'Brien, T. J. 1999, MNRAS, 307, 677

Jenkins, E. B., \& Savage, B. D. 1974, ApJ, 187, 243

Iijima, T. 2006, A\&A, 451, 563

Iijima, T. 2009, A\&A, 505, 287

Iijima, T., \& Esenoglu, H. H. 2003, A\&A, 404, 997

Kerr, F.J., \& Westerhout, G. 1965, in Galactic Structure, ed. A. Blaauw, \& M. Schmidt, The University of Chicago Press, 167

Lynch, D. K., Russell, R. W., Rudy, R. J., Sitko, M., \& Woodward, C. E. 2008, IAU Circ., 8998

Meinel, A. B., Aveni, A. F., \& Stockton, M. W. 1968, Catalog of Emission Lines in Astrophysical Objects, Optical Sciences Center and Steward Obs., The University of Arizona, Tucson

Moore, C.E. 1959, A Multiplet Table of Astrophysical Interest, US Dept. of Commerce, Office of Technical Services, Washington DC

Munari, U., Siviero, A., Valisa, P., et al. 2008, CBET, 1431

Münch, G. 1968, in Nebulae and Interstellar Matter, ed. B. M. Middlehurst, \& L. H. Aller (The University of Chicago Press), 365

Nakano, S. 2008, IAU Circ., 8927

Netzer, H. 1975, MNRAS, 171, 395

Nogami, D., Kuriyama, J., \& Iwata, I. 2008, IAU Circ., 8927

Osterbrock, D. E. 1989, Astrophysics of Gaseous Nebulae and Active Galactic Nuclei (Mill Valley, CA: University Science Books)

Peimbert, M., \& Torres-Peimbert, S. 1987, Rev. Mex. Astron. Astrofis., 14, 540

Rudy, R. J., Russell, R. W., Lynch, D. K., \& Woodward, C. E. 2008, IAU Circ., 8936

Schwarz, G. J., Osborne, J. P., Page, K. L., et al. 2009, ATel, 2157

Seaton, M. J. 1975, MNRAS, 170, 475 


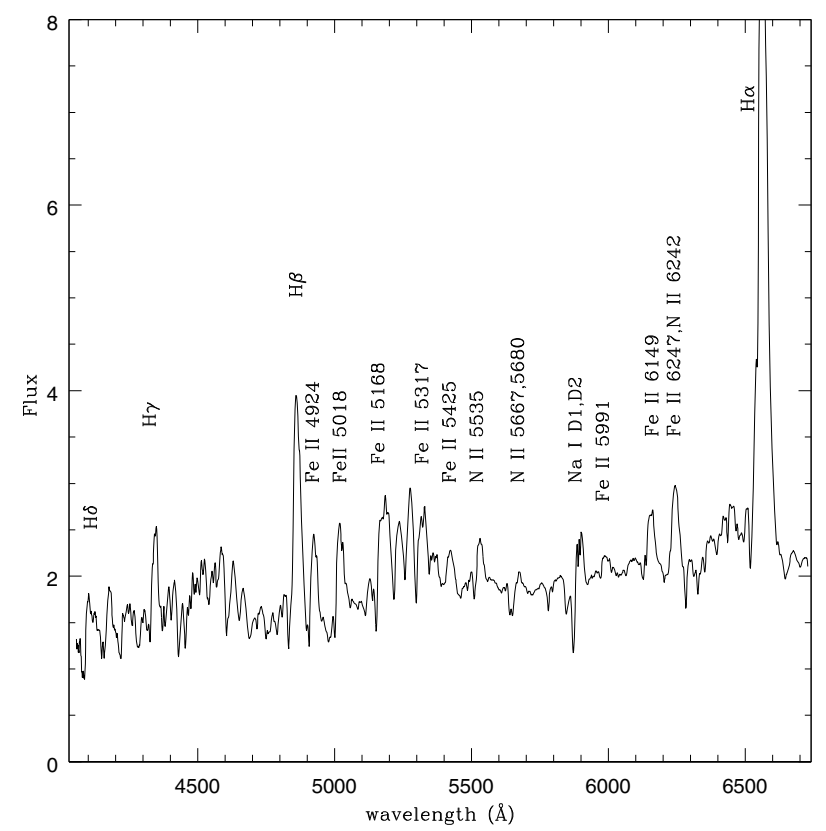

Fig. 4. A spectrum of V2468 Cyg on 2008 March 11. The unit of the ordinate is $10^{-12} \mathrm{erg} \mathrm{cm}^{-2} \mathrm{~s}^{-1} \AA^{-1}$.

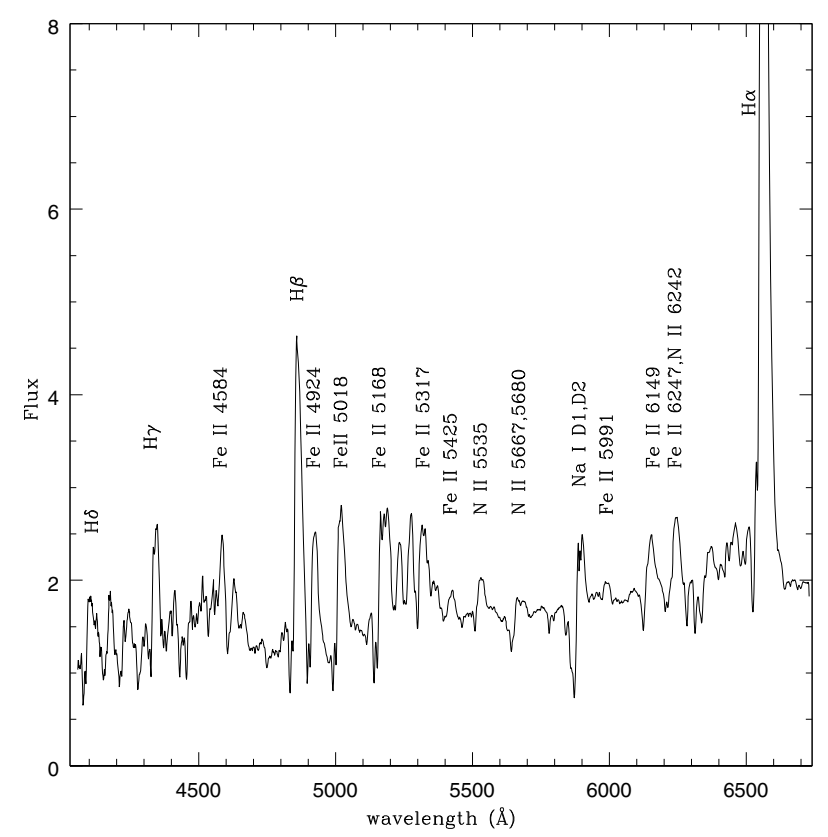

Fig. 6. A spectrum of V2468 Cyg on 2008 March 12.8. The unit of the ordinate is $10^{-12} \mathrm{erg} \mathrm{cm}^{-2} \mathrm{~s}^{-1} \AA^{-1}$.

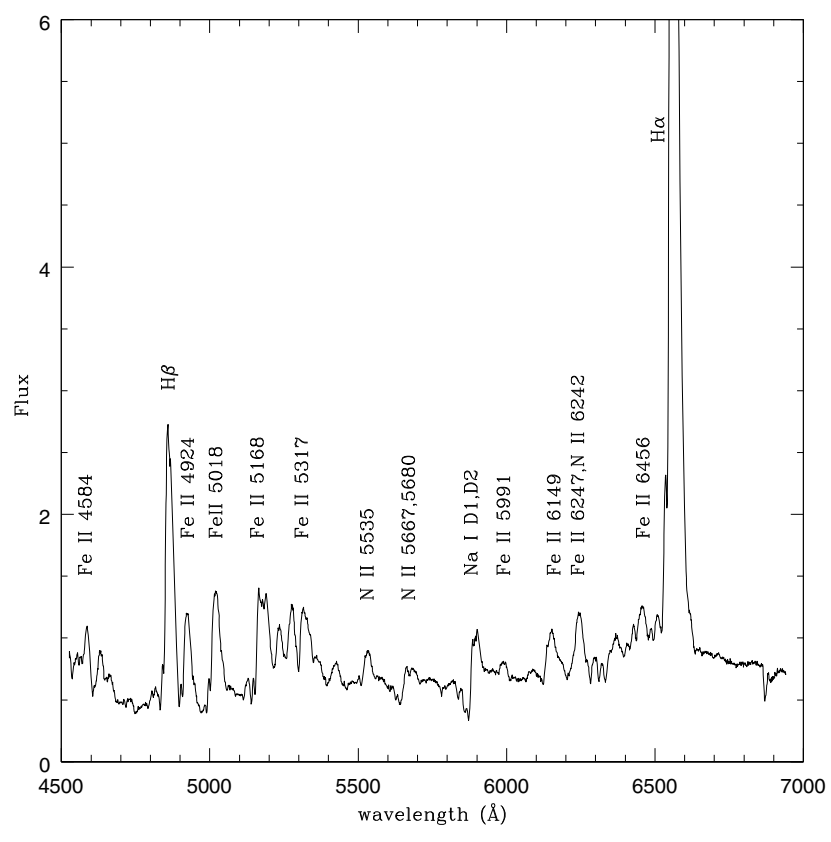

Fig. 7. A spectrum of V2468 Cyg on 2008 March 13. The unit of the ordinate is $10^{-12} \mathrm{erg} \mathrm{cm}^{-2} \mathrm{~s}^{-1} \AA^{-1}$.

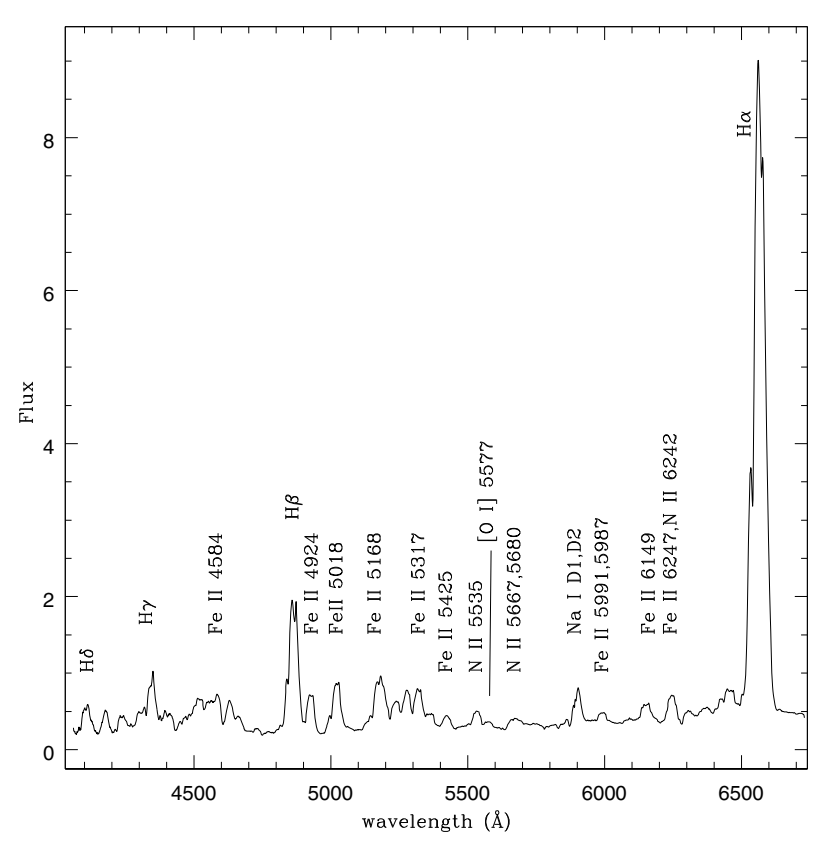

Fig. 8. A spectrum of V2468 Cyg on 2008 March 15. The unit of the ordinate is $10^{-12} \mathrm{erg} \mathrm{cm}^{-2} \mathrm{~s}^{-1} \AA^{-1}$. 
T. Iijima and H. Naito : Spectral evolution of the nova V2468 Cygni

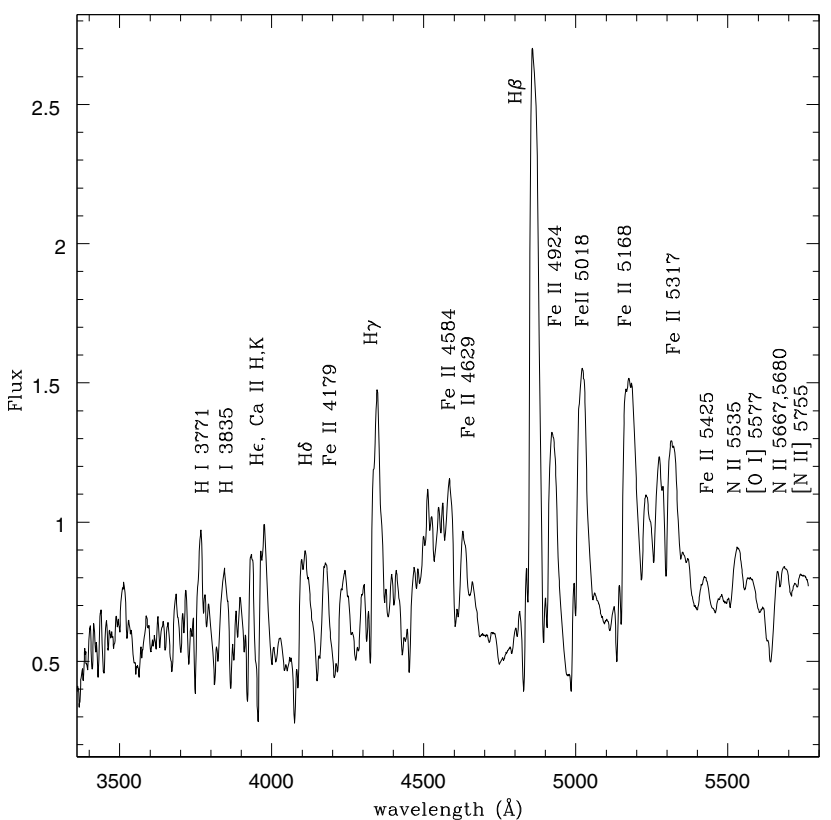

Fig. 9. A spectrum of V2468 Cyg on 2008 March 18. The unit of the ordinate is $10^{-12} \mathrm{erg} \mathrm{cm}^{-2} \mathrm{~s}^{-1} \AA^{-1}$.

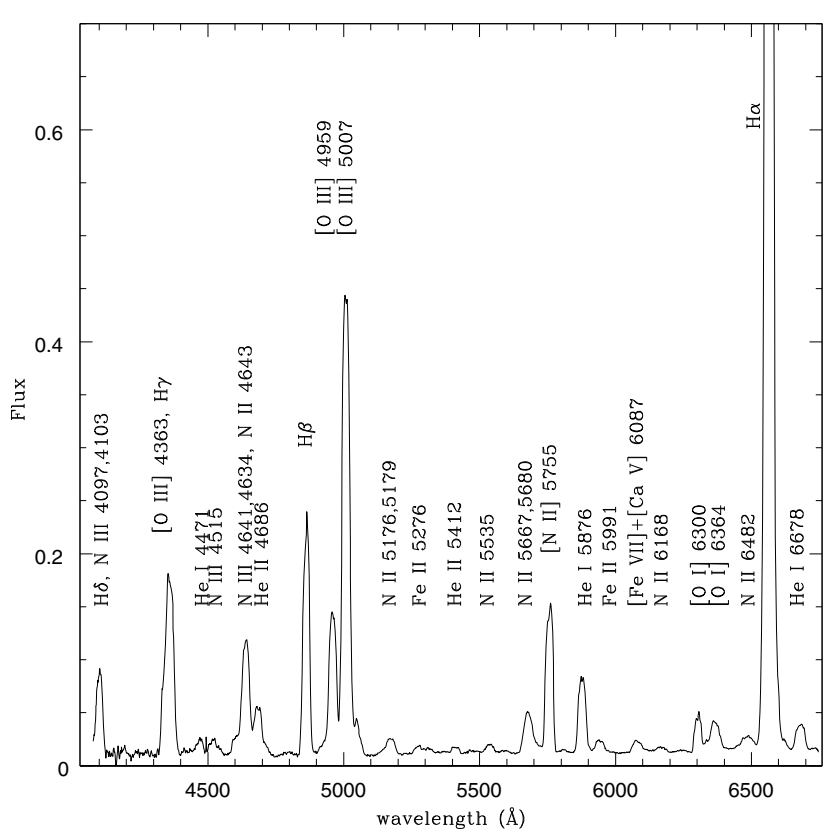

Fig. 13. A spectrum of V2468 Cyg on 2008 July 24. The unit of the ordinate is $10^{-12} \mathrm{erg} \mathrm{cm}^{-2} \mathrm{~s}^{-1} \AA^{-1}$.

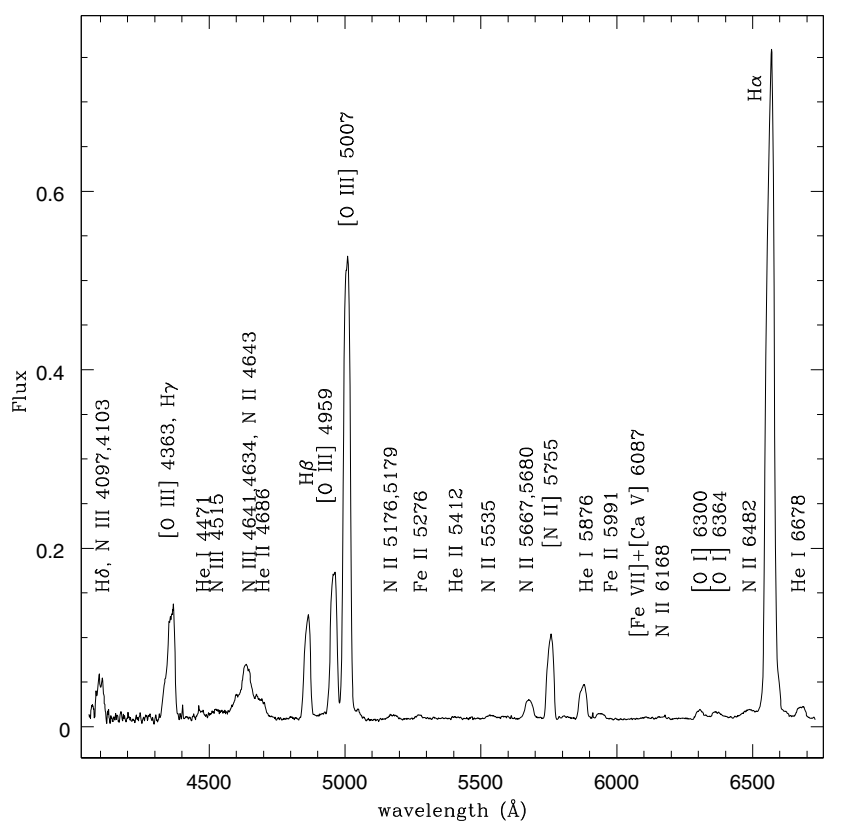

Fig. 15. A spectrum of V2468 Cyg on 2008 November 13. The unit of the ordinate is $10^{-12} \mathrm{erg} \mathrm{cm}^{-2} \mathrm{~s}^{-1} \AA^{-1}$.

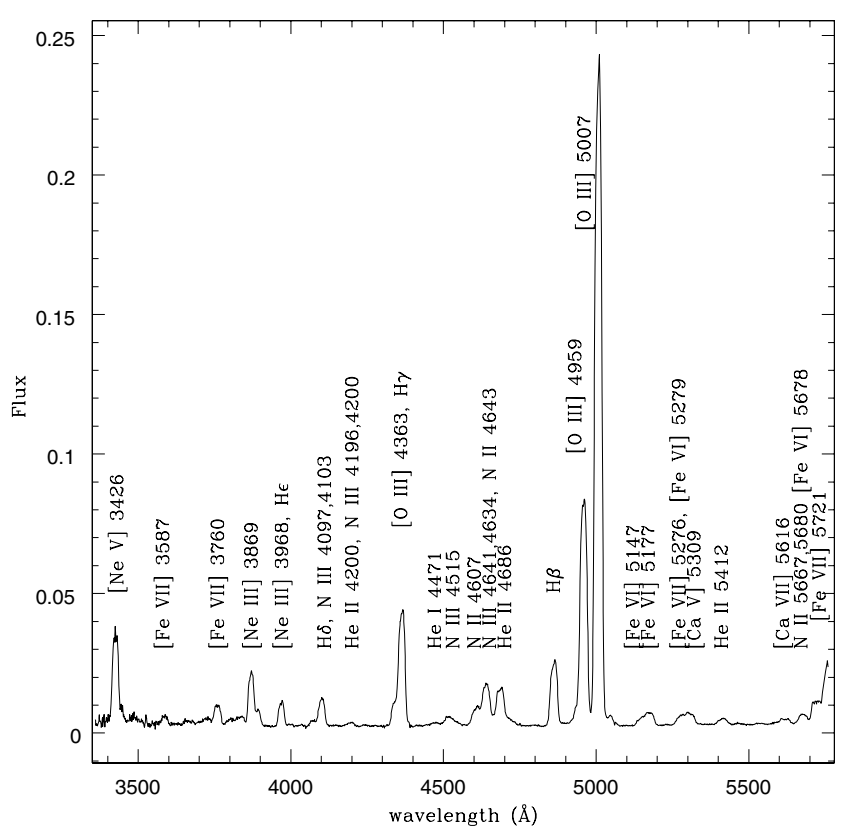

Fig. 16. A spectrum of V2468 Cyg on 2009 June 3. The unit of the ordinate is $10^{-12} \mathrm{erg} \mathrm{cm}^{-2} \mathrm{~s}^{-1} \AA^{-1}$. 


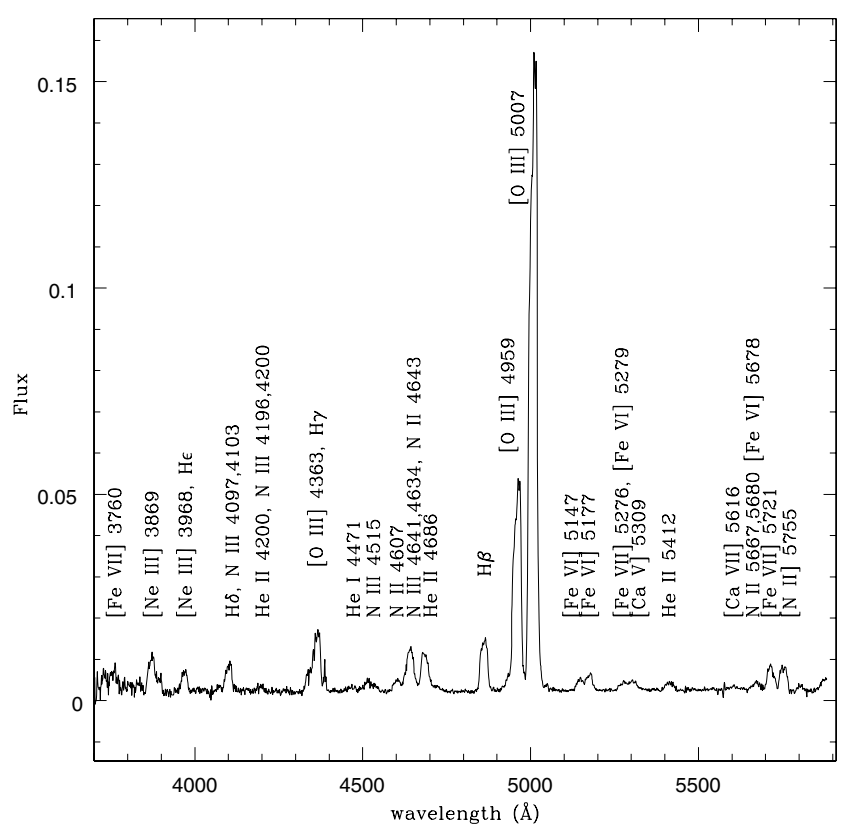

Fig. 20. A spectrum of V2468 Cyg on 2009 October 15. The unit of the ordinate is $10^{-12} \mathrm{erg} \mathrm{cm}^{-2} \mathrm{~s}^{-1} \AA^{-1}$.

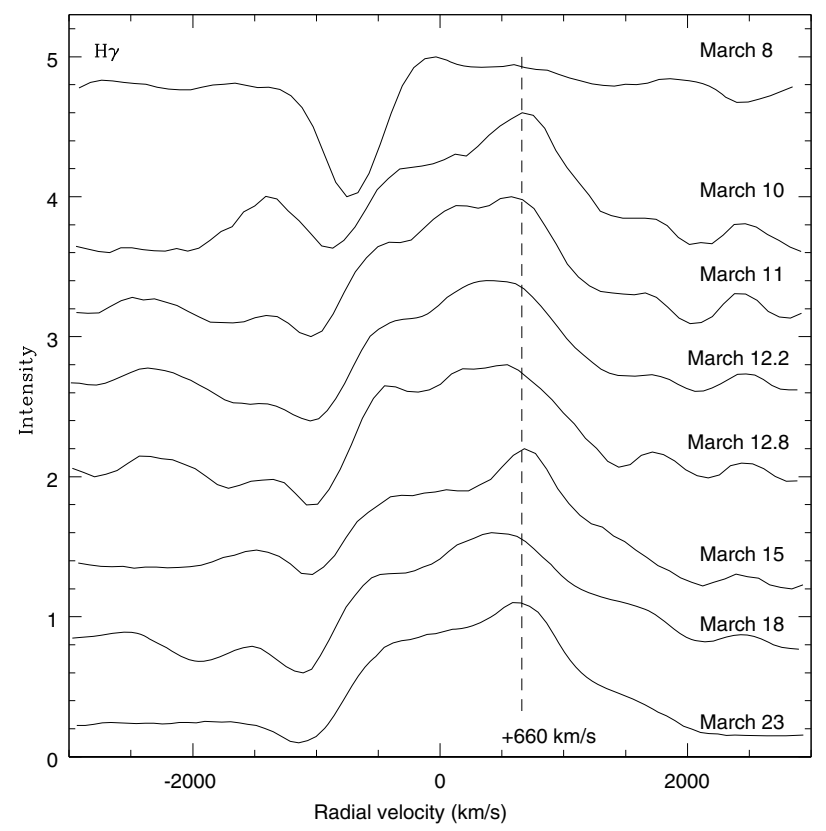

Fig. 23. Profiles of $\mathrm{H} \gamma$ in the early decline stage. The position of the heliocentric radial velocity $+660 \mathrm{~km} \mathrm{~s}^{-1}$ is indicated by a broken line.

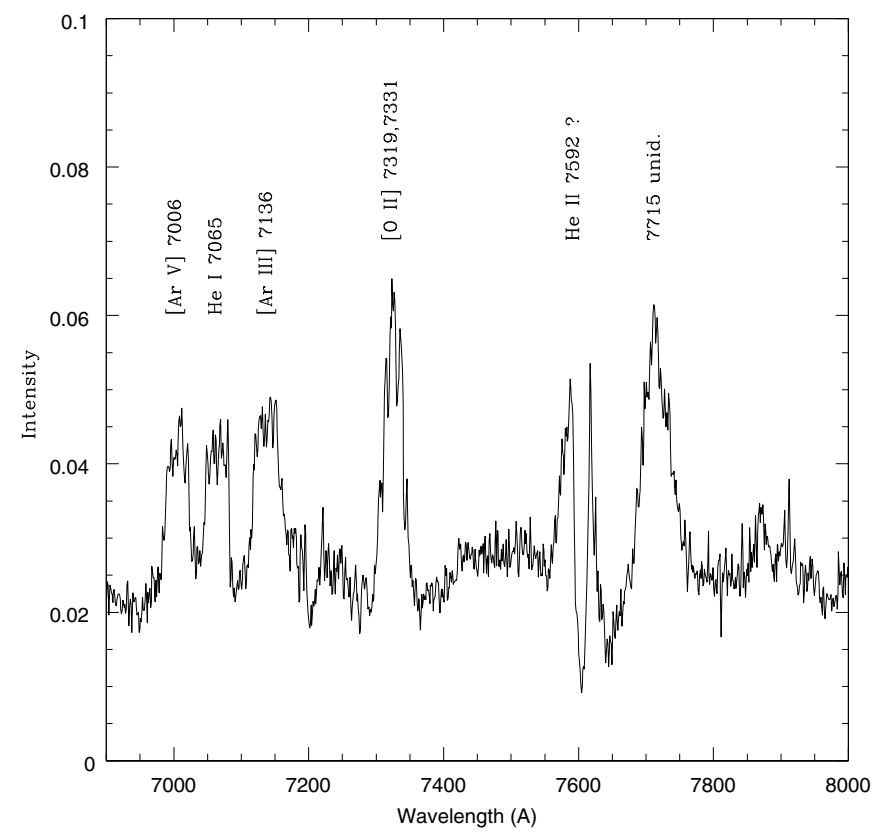

Fig. 31. The red part of the spectrum of V2468 Cyg on 2009 Sept. 27. The unit of the ordinate is $10^{-12} \mathrm{erg} \mathrm{cm}^{-2} \mathrm{~s}^{-1} \AA^{-1}$. 